\title{
Novel lipoproteoplex delivers Keap1 siRNA based gene therapy to accelerate diabetic wound healing
}

Authors: Piul S. Rabbani ${ }^{1 \S}$, Anna Zhou ${ }^{1 \S}$, Zachary M. Borab ${ }^{1}$, Joseph A. Frezzo ${ }^{2}$, Nikita Srivastava ${ }^{2}$, Haresh T. More ${ }^{2}$, William J. Rifkin ${ }^{1}$, Joshua A. David ${ }^{1}$, Samuel J. Berens ${ }^{2}$, Raymond Chen ${ }^{2}$, Sophia Hameedi $^{1}$, Muhammad H. Junejo ${ }^{1}$, Camille Kim ${ }^{1}$, Rita A. Sartor ${ }^{1}$, Che F. Liu ${ }^{2}$, Pierre B. Saadeh ${ }^{1}$, Jin K. Montclare $^{2,3}$, Daniel J. Ceradini ${ }^{1 *}$

\begin{abstract}
Affiliations:
${ }^{1}$ New York University School of Medicine, Hansjörg Wyss Department of Plastic and Reconstructive Surgery, 430 East $29^{\text {th }}$ Street, New York, New York, 10016, United States

${ }^{2}$ New York University Tandon School of Engineering, Chemical and Biomolecular Engineering Department, 6 Metrotech Center, Brooklyn, NY 11201, United States
\end{abstract}

${ }^{3}$ New York University, Chemistry Department, 100 Washington Square East, New York, NY 10003, United States

$\S$ These authors contributed equally to this study

*Corresponding Author: Daniel J. Ceradini, Hansjörg Wyss Department of Plastic and Reconstructive Surgery, New York University School of Medicine, 430 East $29^{\text {th }}$ Street, Alexandria West Tower, $6^{\text {th }}$ Floor- Room 660D, New York, New York, 10016. Phone: (212) 263-8745; Fax: (212) 263-8492; Email: Daniel.Ceradini@nyumc.org 
Funding: This work was supported by the American Diabetes Association "Pathway to Stop Diabetes" to D.C. [grant number 1-16-ACE-08], the NYU Applied Research Support Fund to P.R., and the National Science Foundation to J.K.M. [grant numbers DMR-1505214, IIP-144983].

Running Head: Lipoproteoplex vehicle for diabetic wound therapy 


\begin{abstract}
Therapeutics utilizing siRNA are currently limited by the availability of safe and effective delivery systems. Cutaneous diseases, specifically ones with significant genetic components are ideal candidates for topical siRNA based therapy but the anatomical structure of skin presents a considerable hurdle. Here, we optimized a novel liposome and protein hybrid nanoparticle delivery system for the topical treatment of diabetic wounds with severe oxidative stress. We utilized a cationic lipid nanoparticle (CLN) composed of 1,2-dioleoyl-3-trimethylammonium-propane (DOTAP) and the edge activator sodium cholate $(\mathrm{NaChol})$, in a 6:1 ratio of DOTAP:NaChol (DNC). Addition of a cationic engineered supercharged coiled-coil protein (CSP) in a 10:1:1 ratio of DNC:CSP:siRNA produced a stable lipoproteoplex (LPP) nanoparticle, with optimal siRNA complexation, minimal cytotoxicity, and increased transfection efficacy. In a humanized murine diabetic wound healing model, our optimized LPP formulation successfully delivered siRNA targeted against Keapl, key repressor of Nrf2 which is a central regulator of redox mechanisms. Application of LPP complexing siKeap1 restored Nrf2 antioxidant function, accelerated diabetic tissue regeneration, and augmented reduction-oxidation homeostasis in the wound environment. Our topical LPP delivery system can readily be translated into clinical use for the treatment of diabetic wounds and can be extended to other cutaneous diseases with genetic components.
\end{abstract}

Keywords: siRNA, lipoproteoplex vehicle, Nrf2, Keap1, diabetic wound, gene therapy 


\section{Introduction}

In the United States, nearly 29.1 million people are affected by diabetes and $15 \%$ of these patients suffer from chronic ulcers [1]. These non-healing wounds (i.e. chronic ulcers) are challenging to adequately cover with soft tissue, and represent a leading cause of lower extremity amputations [1]. Diabetic wounds are multifactorial in origin, and increased inflammation, generation of reactive oxygen species (ROS), decreased angiogenesis, and impaired keratinocyte migration are among the implicated pathophysiological mechanisms [2]. No single therapy has proved sufficient and multifaceted therapeutic approaches may be necessary to restore wound healing in the diabetic population. The superficial location and accessibility of these chronic ulcers and wound beds provide a unique opportunity to develop novel vehicles that can deliver their pharmacological drug cargo directly to the area of pathology via topical routes.

RNA interference (RNAi) is a biologically conserved process of sequence specific post-transcriptional gene silencing, and has enormous potential for treating diseases with identified genetic dysregulation and pathology. In RNAi, native double stranded (ds)RNA that has been processed into 21-23 nucleotide long fragments associate with RNA-induced silencing complexes in the cell cytoplasm, to hybridize with and induce cleavage of complementary mRNA sequences [3]. Based on this mechanism, synthetic short interfering RNA (siRNA) are designed to target and degrade specific mRNA transcripts for research and therapeutic purposes. Early pre-clinical studies utilizing siRNA to treat various diseases have been promising, but initial clinical trials failed to impress, and were terminated due to poor efficacy or substantial off-target effects [4]. RNAi technology needs further refinement before widespread clinical use becomes a reality. 
One of the main barriers to successful siRNA therapy is effective delivery of siRNA into cells. The 21-23 base pair siRNA are susceptible to degradation by nucleases and are not readily taken up by cells [4]. Additionally, electrostatic interactions between the negatively charged cellular membrane and negatively charged siRNA do not favor siRNA uptake. To address these issues, a wide array of delivery vehicles have been tested, including liposome based nanoparticles, cationic dendrimers, cyclodextrin polymers, polyethyleneimine, mesoporous silica nanoparticles, protein or peptide based nanoparticles, nucleic acid aptamer based nanoparticles, and bacteriophage packaging RNA based nanoparticles. Delivery routes have been similarly diverse, and include intravascular infusion, injection, inhalation, and topical administration [4]. These delivery systems have achieved varying degrees of efficacy, with eye drops, injectable, and intravenous formulations currently at the forefront of testing for clinical use [4]. However, topical siRNA delivery through the outermost barrier of the stratum corneum remains challenging.

Cationic lipid nanoparticles (CLN) have been studied widely for systemic siRNA delivery and have also shown promise in topical applications [5, 6]. Our lab previously tested 1,2-dioleoyl-3trimethylammonium-propane (DOTAP) and edge activator sodium cholate (NaChol) to form DOTAP $\mathrm{NaChol} \mathrm{(DNC)} \mathrm{for} \mathrm{topical} \mathrm{transdermal} \mathrm{delivery} \mathrm{of} \mathrm{siRNA} \mathrm{[7].} \mathrm{However,} \mathrm{with} \mathrm{a} \mathrm{primarily} \mathrm{lipid} \mathrm{based}$ vehicle formulation, effective siRNA delivery remains suboptimal. Our lab and others have recently designed hybrid vehicles that combine DNC with cationic peptide-based ternary complexes, to enhance nucleic acid complexation and increase transfection efficacy [8-10]. We designed a supercharged coiledcoil protein (CSP), derived from the naturally occurring coiled-coil domain of cartilage oligomeric matrix protein (COMPcc). Like its parent molecule, CSP is a coiled-coil pentamer of alpha helices and has been engineered by site-directed mutagenesis to exhibit positively charged arginine residues at solvent exposed sites. This mutant protein can then enable charge-charge interactions between the cationic guanidium groups of arginine and the anionic phosphate groups of nucleic acids, such as siRNA [10]. When combined with a commercial transfection reagent, CSP has shown the capacity for nucleic acid 
complexation and significant improvement in efficacy in comparison to transfection reagent alone [10]. Other supercharged proteins, that can complex nucleic acids, have shown improvements in in vivo delivery $[10,11]$. Additionally, in CSP, the assembly of its monomeric alpha helical units into a coiledcoil pentamer results in a hydrophobic pore capable of encapsulating several small molecule drugs, and provides the possibility for co-delivery of a pharmaceutical drug in combination with siRNA [10, 12]. The next phase in design and our resulting vehicle, therefore, combined CSP with a CLN (DNC) for improved siRNA delivery into mammalian tissues, such as skin.

Pharmacological therapies currently play a limited role in the management of diabetic wounds. Diabetes and its associated complications pose a significant economic and social burden, accounting for up to \$245 billion in health care costs in 2012 [1]. Despite the advances made in understanding the underlying pathophysiology, treatment of diabetic wounds remains challenging [13]. The mainstay of wound care includes dressings, negative pressure wound therapy, surgical debridement, and local tissue rearrangement, rather than the targeting of specific abnormalities caused by the hyperglycemic state [14, 15]. Previous work investigating wound healing demonstrated increased levels of ROS in diabetic cutaneous wounds compared to normoglycemic wounds, suggesting an imbalance in reduction-oxidation (redox) homeostasis pathways and a potential therapeutic target [16-20].

To investigate and correct this ROS imbalance, our lab previously targeted a central regulator of antioxidant pathways, the transcription factor, nuclear factor erythroid-2 like 2 (Nrf2) and its repressor, Kelch like ECH-associated protein 1 (Keap1) [20]. In the absence of oxidative stress, Nrf2 is bound to Keap1 in the cytoplasm, targeted for ubiquitination, and subsequent proteasomal degradation. In the presence of oxidative stress, certain cysteine-rich oxidant and electrophile sensing regions of Keap1 are covalently modified, which prevent the ubiquitination of Nrf2. Nrf2 then dissociates from its repressor, Keap1, and translocates from the cytoplasm into the nucleus. There, Nrf2 binds antioxidant response 
elements in the promoter region of a wide array of genes involved in protection against oxidative stress, protein stability, proteasome integrity, autophagy, senescence, and inflammation [21]. In diabetes, chronic hyperglycemia induces a phenotype of poor wound healing, characterized in part by an unbalanced production of ROS. Atypical upregulation of Keap1 in diabetes and subsequent degradation of Nrf2 implies a lack of antioxidant mechanisms. We have shown that pharmacologically activating Nrf2 normalizes the oxidative state of diabetic wounds and consequently, accelerates wound closure in diabetic mice [20]. Lipofectamine, a commercial transfection reagent, has been used to display proof of concept for siRNA delivery, but is not appropriate for human use [20, 22, 23]. Our goal is to develop an efficient delivery vehicle that is safe for humans and can be rapidly translated to the clinic.

In this study, we develop a novel, precisely engineered LPP vehicle consisting of DNC cationic liposomes and CSP protein for enhanced topical delivery of siRNA. We hypothesized that use of this novel LPP system to efficiently deliver complexed Keapl siRNA, will activate Nrf2-mediated endogenous antioxidant mechanisms, normalize the ROS imbalance, and normalize tissue regeneration in murine diabetic wounds.

\section{Materials and Methods}

\subsection{Materials}

Lipid nanoparticles consisted of DOTAP (Avanti Lipids, Alabaster, AL) and NaChol (Sigma Aldrich, St. Louis MO) in 6:1 w/w ratio. 4.5g/L DMEM, Opti-MEM, Lipofectamine 2000, FBS, trypsin, penicillin streptomycin (P/S) solution, and siRNA were purchased from Thermo Fisher Scientific, (Waltham, MA). CSP was expressed and purified as described previously [10]. 


\subsection{Mice}

All animal protocols were approved by the New York University Langone Medical Center Institutional Animal Care and Use Committee. Male diabetic Lepr ${ }^{\mathrm{db} / \mathrm{db}}$ mice $(\mathrm{db} / \mathrm{db})$ and non-diabetic mice C57BL/6J (WT, +/+), aged 12 weeks, were obtained from Jackson Laboratories (Bar Harbor, ME). Hyperglycemic status of $\mathrm{db} / \mathrm{db}$ mice was confirmed with two separate random blood glucose measurements of $>400$ $\mathrm{mg} / \mathrm{dL}$. Mice were housed in a temperature-controlled, virus-free barrier animal facility with a 12-hour light/dark cycle and maintained on chow diet/water ad libitum.

\subsection{LPP preparation}

1,2-dioleoyl-3-trimethylammonium-propone (DOTAP) stock was provided by the manufacturer (Avanti Lipids) as a chloride salt, dissolved in chloroform. Sodium cholate ( $\mathrm{NaChol})$ stock was provided by the manufacturer (Sigma-Aldrich) as a solid particulate and dissolved in methanol. DOTAP:NaChol stocks, mixed in $2: 1 \mathrm{v} / \mathrm{v}$ chloroform methanol mixtures at predetermined $\mathrm{w} / \mathrm{w}$ ratios, were subjected to a constant stream of $\mathrm{N}_{2}$ gas until total evaporation ( $\sim 1$ hour) followed by vacuum dessication (3 hours). The thin film lipid mixture was then hydrated with double filtered deionized water, lightly vortexed to resuspend the mixture into large multilamellar vesicles (LMV) and placed in a bath sonicator at $25{ }^{\circ} \mathrm{C}$ for 15 minutes. The hydrated LMV mixture was then extruded through a stack of three $200 \mathrm{~nm}$ polycarbonate filters 21 times fitted into a LiposoFast LF-50 extruder at a constant pressure ( $<600 \mathrm{psi}, 41$ bar) to produce small unilamellar DOTAP:NaChol liposomes (DNC). Stock siRNA were diluted to $20 \mu \mathrm{M}$ and CSP $(7.2 \mathrm{kDa} / \mathrm{mol})$ was prepared at $0.98 \mathrm{mg} / \mathrm{mL}$. siRNA was added dropwise to CSP in the desired weight ratios, and molecular grade water was added to obtain equal CSP and siRNA volumes. This mixture was gently hand vortexed and incubated at room temperature for 30 minutes to allow siRNA to complex with CSP. During incubation, DNC prepared in a 6:1 DOTAP:NaChol w/w ratio $(0.84 \mu \mathrm{g}$ of DOTAP/ $\mu \mathrm{l}$ ) was diluted in HEPES to equal the volume of siRNA:CSP mixture and incubated at room temperature for 10-15 minutes. The siRNA:CSP mixture was then added dropwise to the diluted DNC 
and incubated for another 15 minutes at room temperature. The final LPP was used within 30 minutes. All LPP formulations are noted in DNC:CSP:siRNA in a w/w/w ratio.

\subsection{Electrophoretic mobility shift assay}

LPPs were prepared as described above, in varying ratios. For comparison, complexes without either CSP or DNC were prepared with water in lieu of omitted components. Following the final 15-minute incubation, fully formed LPP with loading dye was loaded onto a $2 \%$ agarose gel. Naked siRNA and a nucleotide ladder were used to mark the position of the siRNA.

\subsection{Size and zeta potential characterization of LPP}

Zeta potential and dynamic light scattering (DLS) size analyses of protein, siRNA, and complex were determined using Zetasizer Nano ZS90 (Malvern Instruments, UK) with a $630 \mathrm{~nm}$ laser source with the applied settings: material liposome (refractive index 1.000), absorption 0.001 , dispersant water with a viscosity of $0.890 \mathrm{cP}$ and refractive index of 1.000 . Zeta potential was calculated based on the measured electrophoretic mobility and fit into a Smoluchowski equation [24]. Prepared LPPs were diluted to $3 \mathrm{~mL}$. Per sample, three measurements were taken where each measurement consisted of 20 runs. Data is represented as mean \pm standard deviation $\mathrm{mV}$ and $\mathrm{z}$-average of 3 trials.

Transmission electron microscopy (TEM) of the DNC alone (10:0:0), with siRNA (10:0:1) and with siRNA and CSP (10:1:1) was used to analyze the morphology. Complexes were formed as described above and $5 \mu \mathrm{L}$ of each sample was applied to a glow-discharged carbon coated 400 mesh copper grid and incubated for 30 seconds and blotted with Whatman filter paper. A $5 \mu \mathrm{L}$ solution of negative stain uranyl acetate $\left(1 \%\right.$ w/v in $\left.\mathrm{H}_{2} \mathrm{O}\right)$ was applied and quickly blotted twice before a final $5 \mu \mathrm{L}$ application of negative stain for 5 minutes. After this period, the stain was blotted and the grid was immediately imaged using a Phillips CM-12 transmission electron microscope equipped with a with Gatan 4k x 2.7k digital camera. The sizes of DNC liposomes were measured using ImageJ (NIH, Bethesda, MD). 
2.6. In vitro transfection studies

Murine NIH-3T3 fibroblasts (CRL-1658, ATCC, Manassas, VA) were maintained in DMEM/10\% $\mathrm{FBS} / 1 \% \mathrm{P} / \mathrm{S}$, cultured in $37^{\circ} \mathrm{C}, 5 \% \mathrm{CO}_{2}$. $3 \mathrm{~T} 3$ cells were seeded in 24 -well plates at a density of 40,000 cells/well in $500 \mu \mathrm{L}$ DMEM/ 10\% FBS/ 1\% P/S for 70-90\% confluency. Cells were incubated overnight and starved for 6 hours in $500 \mu \mathrm{L}$ DMEM/ 0.5\% FBS without P/S. 20 pmol of nonsense siRNA (AM4635), siKeap1 (siRNA mouse Silencer select Keap1 ID:s78526), or siGAPDH (AM4624) were added to appropriate amounts of CSP and DNC as dictated by chosen w/w/w ratios and were added dropwise to cells in $500 \mu \mathrm{L}$ DMEM without FBS or $\mathrm{P} / \mathrm{S}$, keeping the final volume constant. Lipofectamine 2000 was mixed with OptiMEM and combined with siRNA as a positive control. After 3.5 hours of transfection, 10\% FBS was added back to the cells. Following an overnight incubation, media was changed to $500 \mu \mathrm{L}$ DMEM/ $10 \% \mathrm{FBS} / 1 \% \mathrm{P} / \mathrm{S}$. Cells were harvested after 24 hours for RNA isolation and after 48 to 72 hours for nuclear and cytoplasmic protein isolation.

\subsection{Quantitative RT-PCR}

Total RNA was isolated using the RNeasy Tissue Mini Kit (Qiagen, Valencia, CA) according to the manufacturer's instructions. Quality of mRNA was assessed using a Nanodrop-1000 Spectrophotometer (Thermo Fisher Scientific, Waltham, MA). $500 \mathrm{ng}$ of total RNA was reverse transcribed using the HighCapacity cDNA synthesis kit (Applied Biosystems, Foster City, CA). mRNA quantification was determined by real-time quantitative RT-PCR using a SYBR green detector (Life Technologies, NY) with a QuantStudio 7 Flex (Applied Biosystems). Relative mRNA expression was calculated using standard curve method.

\subsection{Cell cytotoxicity assay}

Cytotoxicity was assessed using the CytoTox 96 Non-Radioactive Cytotoxicity Assay (Promega, Madison, WI) per manufacturer's instructions. Briefly, murine NIH-3T3 fibroblasts were plated in a 96well plate and treated with various ratios of CSP, DNC, or DNC:CSP. Damage to cell membranes results 
in release of cytosolic lactate dehydrogenase (LDH) into cell culture media. Following 24 hours of transfection, aliquots of supernatant were transferred into a 96-well flat bottom enzymatic assay plate. LDH levels were quantitatively measured using a coupled enzymatic assay, which converts the tetrazolium salt (iodonitro tetrazolium violet) into a red formazan product in the presence of LDH. Absorbance at 490-492 nm was read using a standard 96 well plate reader (SpectraMAX 340, Molecular Devices, Sunnyvale, CA), and the percentages of cell cytotoxicity and cell survival were calculated. Untreated cells were used as a standard for cell survival.

\subsection{In vitro ROS production assay}

The production of free radicals was assessed by using chloromethyl-2'7'-dichlorofluoroscein diacetate (CM- $\mathrm{H}_{2}$ DCFDA) technique [25]. Murine NIH-3T3 fibroblasts were seeded in a 6-well plate at $2 \times 10^{4}$ cells $/ \mathrm{cm}^{2}$ and treated with either lipoplex or lipoproteoplex with siKeapl or siNonsense. The CM$\mathrm{H}_{2}$ DCFDA assay was performed 16 hours after transfection. Briefly, $10 \mu \mathrm{M}$ of DCFDA was added to trypsinized cells and incubated for 45 minutes at $37^{\circ} \mathrm{C}, 5 \% \mathrm{CO}_{2}$. Following a wash with PBS, cells were analyzed for fluorescence using a FACS Calibur (BD Biosciences, San Jose, CA). Accumulation of the oxidized derivative, DCF, was measured at 492-495/517-527 nm excitation/emission.

\subsection{Cutaneous wound model}

We utilized a humanized stented-wound healing model described previously [26]. Animals were age matched to 12 weeks, and blood glucose was confirmed to be $>400 \mathrm{mg} / \mathrm{dL}$ at the time of wounding. Animals were anesthetized, shaved, and hair removed with Nair (Nairß, Church and Dwight, Co., Princeton, NJ). Wounding sites on mice dorsum were swabbed with povidine iodine solution and a10 $\mathrm{mm}$ punch biopsy tool and surgical scissors were used to create two circular, full-thickness cutaneous wounds extending through the panniculus carnosus. A donut-shaped stent made of $0.5 \mathrm{~mm}$ thick silicone (Grace Bio-Laboratories, Bend, OR) with an internal diameter of $10 \mathrm{~mm}$ and outer diameter of $14 \mathrm{~mm}$ was centered on the wound and secured in place with four interrupted 4-0 silk sutures (Ethicon, Inc., 
Somerville, NJ). Intramuscular injections of Buprenex $(0.1 \mathrm{mg} / \mathrm{kg})$ were given postoperatively and animals were monitored daily.

\subsection{In vivo transfection}

24 hours after wounding, $58.5 \mu \mathrm{L}$ of LPP solution was pipetted dropwise to the wound bed within 30 minutes of preparation each day. The LPP solution consisted of 250 pmol of siRNA (siNonsense, siGLO (D-001630-02-05), or siKeap1), with DNC and CSP in optimized ratios.

2.12. Wound closure analysis, histology, mRNA analysis, and western blotting Digital photographs were recorded on day $0,7,10,14,17,19,21,24,27,29,31$ and, until wound closure. Digital photographs were analyzed photometrically for wound closure calculated as percent wound area open, compared to a standardized $78.54 \mathrm{~mm}^{2}$ original wound as marked by the $10 \mathrm{~mm}$ stent inner diameter using Image $\mathbf{J}(\mathrm{NIH}$, Bethesda, MD). Percent excisional wound area as a measure of wound burden was compared to historical C57BL6 wild type controls and area-under-the-curve (AUC) was calculated using the trapezoidal rule in Graphpad Prism (San Diego, CA).

For histology, wounds were excised, bisected, and fixed in $4 \%$ paraformaldehyde at $4{ }^{\circ} \mathrm{C}$ overnight, washed in $1 \mathrm{xPBS} 3$ times, and processed into paraffin. $5 \mu \mathrm{m}$ wound tissue sections were stained with hematoxylin and eosin (H\&E) and CD31 antibody. Slides were scanned and digitized to 40x magnification using Leica SCN400F (Leica Biosystems, Wetzlar, Germany). 10-day wound images were analyzed in Adobe Photoshop (Santa Cruz, CA) for epithelial gap distance, granulation tissue area, and CD31 positive cell count per high power field (hpf).

For mRNA analysis, at the time of sacrifice, the wounds were excised, bisected, and trimmed to only contain treated area. Livers were collected as well. Tissue samples were either flash frozen in liquid nitrogen or used immediately for mRNA harvesting. Tissues were processed with trizol, homogenized 
using polytron tissue homogenizer (Kinematica, Bohemia, NY), and RNA extracted according to the RNeasy Tissue Mini Kit (Qiagen, Valencia, CA).

For western blotting, wounds were excised, and both nuclear and cytoplasmic lysates were prepared. 20 $\mu \mathrm{g}$ lysate was run on 10\% Mini Protean Tetra Cell Gels (Bio-Rad, Hercules, CA) and transferred to a fluorescent polyvinylidene fluoride membrane (EMD Millipore, Billerica, MA). The membrane was blocked for one hour with 5\% milk in Tris-buffered saline with $0.01 \%$ Tween (Pierce, Rockford, IL) and probed with primary antibodies specific for Keap1 (60027-Ig; Proteintech, Chicago, IL), Nrf2 (ab92946; Abcam, Cambridge, MA), PCNA (2586; Cell Signaling Technology, Danvers, MA), Beta actin (ab8229; Abcam). Secondary antibodies were infrared dye goat anti rabbit (926-68071; LI-COR Biosciences, Lincoln, NE) and goat anti mouse (926-32210; LI-COR Biosciences) and membrane was imaged with LICOR CTx and quantified using Image Studio software (LI-COR Biosciences).

\subsection{In vivo ROS quantification}

A luminol-based bioluminescent substrate, L-012 (Wako Chemicals, Richmond, VA) prepared in 1x PBS at $0.5 \mathrm{mg} / 100 \mu \mathrm{L}$, was injected intraperitoneally into anesthetized mice with 6-day-old wounds. The bioluminescence of L-012 upon detecting ROS was measured at 5-minute intervals and captured with Spectrum In Vivo Imaging System (IVIS) (PerkinElmer, Waltham, MA) and quantified using Living Image (PerkinElmer).

2.14. Fluorescent siRNA wound bed penetration and persistence in wound bed Wounded animals were treated using the red fluorescent siGLO in the LPP formulation. For wound bed penetration, animals were sacrificed on days 3,7 , and 10 . Wounds were excised, bisected in a cranialcaudal orientation, fixed overnight in $4 \%$ paraformaldehyde, washed three times in 1xPBS, cryoprotected through sucrose and embedded in OCT. $20 \mu \mathrm{m}$ sections were stained with DAPI and photographed using Olympus BX51 Microscope (Center Valley, PA). 
To study siRNA persistence in the wound bed, any unabsorbed DNC:CSP:siGLO after 2 treatments was removed from the wounds, and fluorescence from siGLO was imaged using the Spectrum IVIS (PerkinElmer, Waltham, MA) over a 7-day course until loss of siGLO signal. Representative photographs were analyzed and fluorescence was quantified on Living Image (PerkinElmer, Waltham, MA).

\subsection{Statistical analysis}

Data are expressed as mean \pm standard deviation for triplicate experiments. Statistical significance between means was analyzed by two-tailed Student's t-test using the GraphPad Prism 5 software (San Diego, CA). $p<0.05$ was considered statistically significant.

\section{Results}

\subsection{LPP complexes with siRNA to form stable nanoparticle}

In order to assess suitability of LPP for packaging siRNA and transfection into mammalian cells, we began by characterizing the biophysical features of the nanoparticle. To determine the combination of DNC:CSP:siRNA for optimal siRNA packaging capacity, we performed electrophoretic mobility shift assays. siRNA was complexed with either DNC or CSP, and then with both. Increasing ratios, by weight, of DNC:siRNA (Fig 1A) and increasing ratios of CSP:siRNA corresponded with decreasing amounts of free uncomplexed siRNA (Fig 1B). Increasing amounts of DNC with constant CSP:siRNA ratio corresponded with decreasing amounts of free uncomplexed siRNA (Fig 1C). We observed a remarkable decrease in uncomplexed siRNA once the DNC:siRNA ratio reached at least 8:1 (Fig 1A), and this

decrease was consistent even with introduction of CSP (Fig 1C). The data strongly suggests that ratios of DNC:siRNA beyond 8:1, in conjunction with near equal weights of CSP and siRNA in the LPP, would maintain similar siRNA complexing patterns. Increasing the amount of DNC to 15 times the weight of siRNA and CSP maintained similar siRNA binding patterns (data not shown). 
Next, we studied the diameter, morphology and surface charge (zeta potential) of LPP variations to assess nanoparticle stability and cellular uptake potential. LPP formulations with varying CSP quantities, 10:0:1, 10:1:1, 10:3:1, and 10:5:1 (DNC:CSP:siRNA) loaded with 20 pmol of siRNA, had diameters of $198.08 \pm$ $9.09 \mathrm{~nm}, 174.22 \pm 8.71 \mathrm{~nm}, 203.00 \pm 10.15 \mathrm{~nm}$, and $304.20 \pm 15.21 \mathrm{~nm}$, respectively (Fig 1D). The 10:1:1 DNC:CSP:siRNA formulation yielded the smallest nanoparticle, with the highest potential for membrane penetration. Zeta potential measurements for the same LPP formulations reveal values of 66.4 $\pm 3.3 \mathrm{mV}, 34.5 \pm 1.7 \mathrm{mV}, 37.1 \pm 1.9 \mathrm{mV}$, and $29.0 \pm 1.5 \mathrm{mV}$, respectively. The addition of CSP lowered the zeta potential, but the 10:1:1 and 10:3:1 DNC:CSP:siRNA formulations formed stable nanoparticles (Fig 1E), each with surface charges above $30 \mathrm{mV}$ [27].

To assess changes in nanoparticle characteristics at different loading doses, we incorporated increasing amounts of siRNA into the LPPs. The weights of the other two components, DNC and CSP, were adjusted accordingly, to adhere to the desired ratio. Liposomes comprised of solely DNC displayed an average diameter of $131.11 \pm 7.33 \mathrm{~nm}$. Adding siRNA loads of 80 pmol, 250 pmol, and 500 pmol with CSP at 10:1:1 DNC:CSP:siRNA significantly increased LPP particle size to $245.13 \pm 46.94 \mathrm{~nm}, 360.55 \pm$ $38.21 \mathrm{~nm}$, and $410.67 \pm 127.75 \mathrm{~nm}$, respectively $(\mathrm{p}<0.05)$ in comparison to DNC alone (Fig $1 \mathrm{~F})$. Among the different siRNA loads, the diameters were not significantly different. Zeta potential values of the 80 pmol, 250 pmol, and 500 pmol siRNA-loaded LPP were $31.50 \pm 0.35 \mathrm{mV}, 38.2 \pm 0.98 \mathrm{mV}$, and $43.90 \pm$ $0.46 \mathrm{mV}$, respectively. These zeta potential values were considerably less than that of only liposome $(58.57 \pm 2.42 \mathrm{mV})$ but all remained above the $30 \mathrm{mV}$ threshold for stability (Fig 1G).

We then analyzed the morphologies of DNC alone, DNC:siRNA (10:1) and DNC:CSP:siRNA (10:1:1) using TEM. The inherent flexibility of DNC is retained upon the addition of siRNA alone and both siRNA and CSP. This wass clearly seen at the edges of several DNC particles where bending occurs without rupture (Figure 2A-C). Based on the TEM micrographs, the diameter measurements of the DNC 
alone at $126.64 \pm 14.67 \mathrm{~nm}$ were most consistent with the DLS results (Fig 2A, D). The TEM-based mean diameter of 10:1 DNC:siRNA was $176.70 \pm 47.83 \mathrm{~nm}$, and that of 10:1:1 DNC:CSP:siRNA was $160.94 \pm$ $34.21 \mathrm{~nm}$, both larger than that of DNC alone. Importantly, the trend in size based on TEM corroborated the DLS data, that CSP addition reduces particle size. The absence of large protein aggregates in the DNC:CSP:siRNA micrographs also suggested either CSP:siRNA coating on the DNC surface, or encapsulation by the DNC. Our LPP characterization data demonstrated that the size, charge, elasticity, and siRNA carrying capacity of the DNC:CSP:siRNA LPP present a plausible candidate for further testing in biological systems.

\subsection{LPP in 10:1:1 DNC:CSP:siRNA attains optimal in vitro transfection}

To analyze in vitro transfection efficacy of various formulations of the DNC:CSP:siRNA LPP, we combined them with 20 pmol siRNA targeted against GAPDH in 3T3 mouse fibroblasts, under low serum conditions. The commercially available cationic lipid carrier, Lipofectamine 2000, served as a positive control. 10:1:1 DNC:CSP:siGAPDH induced the highest knockdown with $62.00 \pm 6.51 \%(\mathrm{p}=0.0001)$ compared to untreated cells, while the 10:0:1 formulation, notably without CSP, only achieved $48.00 \pm$ 8.12\% knockdown (Fig 3A). The difference in gene silencing between the two formulations, 10:1:1 and 10:0:1 DNC:CSP:siGAPDH, is significant as well $(\mathrm{p}<0.05)$. This indicated that liposomes alone could mediate siRNA delivery into cells, but the addition of CSP achieved maximal knockdown, similar to that with Lipofectamine (Fig 3A). Transfection with LPP containing siNonsense (siNS) confirmed that the reduced GAPDH gene expression was a product of specific GAPDH gene silencing instead of nonspecific effects on the cells (Fig 3B). The results of gene silencing efficacy helped identify the 10:1:1 DNC:CSP:siRNA formulation as a suitable candidate for additional studies.

We used both transcriptional and translational analysis of Keap1 and Nrf2 to determine the time points most suitable for assessing knockdown efficiency of LPP-delivered siKeapl. To model diabetic 
hyperglycemia, we cultured 3T3 fibroblasts in $25 \mathrm{mM}$ glucose DMEM instead of $5 \mathrm{mM}$ glucose DMEM used for normoglycemic conditions [28]. Consistent with the results from Fig 3A, Keapl mRNA knockdown was observed at 24 hours post-transfection with the 10:1:1 formulation and remained consistent through 72 hours post-transfection. Transfection with Nonsense siRNA (siNS) reduced Keapl expression slightly. Nevertheless, siKeapl knocked down gene expression significantly, in comparison to both siNS-transfected and untreated cells (Fig 3C). The gene expression correlated with decreased cytoplasmic Keap1 protein expression at 48 hours post-transfection (Fig 3D). Since Keap1 normally inhibits Nrf2 stability and translocation into the nucleus, we examined nuclear Nrf2 protein levels at 0 , 48, and 72 hours post-transfection. Nrf2 was virtually undetectable within the nucleus at 0 hours, as expected. At 48 hours post-transfection with siKeapl, we observed an increase in Nrf2 band intensity. The upregulation of Nrf2 was maintained until the 72-hour time point post-transfection (Fig 3E). Successful knockdown of Keapl gene expression and protein expression confirmed the efficacy of the LPP vehicle. The increase in nuclear Nrf2 protein expression for at least 72 hours will be essential in identifying ideal assessment time points for future studies.

We wanted to evaluate whether the Nrf2 nuclear localization induced by transfection with the 10:1:1 DNC:CSP:siRNA is sufficient for functional relevance. As Nrf2 transcribes antioxidant genes, we assessed intracellular ROS following transfection. Fibroblasts cultured in hyperglycemia were transfected with siKeaplwith 10:1:1 DNC:CSP:siKeapl, and then ROS quantified using the fluorescent oxidative stress indicator, CM-H $\mathrm{H}_{2}$ DCDFA. Keapl knockdown with 10:1:1 DNC:CSP:siKeapl reduced lower intracellular ROS, notably different from the ROS content of cells transfected with 10:1:1 DNC:CSP:siNS, 10:1 DNC:siKeap1, or DNC:siNS (Fig 3F).

We assessed cytotoxicity upon in vitro transfection to further evaluate the applicability of the 10:1:1 LPP and determine whether the LPP compromises cell integrity. Various ratios of DNC:CSP:siKeapl were 
evaluated in 3T3 cells. This assay revealed that increasing amounts of DNC, with and without CSP, did not result in significant cell damage (Fig 3G). In an additional assay, we were also able to visualize red fluorescent siGLO delivered with 10:1:1 LPP past the cell membrane and within the cell (Fig 3H). Based on the biophysical data, in vitro transfection efficiencies, and cytotoxicity data, we chose the ratio of 10:1:1 DNC:CSP:siRNA as the LPP delivery vehicle for all subsequent in vivo studies, and it will be abbreviated as LPP-10 in the remainder of the text.

3.3. LPP delivered siRNA penetrates to subcutaneous tissue and persists in wound bed To assess depth of penetration of LPP-delivered siRNA, we incorporated a red fluorescent siRNA, siGLO, into LPP-10, and applied siGLO or siNS to standardized mouse wounds. We harvested and analyzed wounds at post-excision days 3, 7, and 10. Through analysis of wound tissue sections, we found that the siGLO-Red permeated beyond the subcutaneous adipose tissue and reached the panniculus carnosus muscle layer by day 10 . The siGLO treatment remained in the wound bed environment with minimal lateral diffusion, as shown in the representative wound edge sections (Fig 4A). To assess siRNA persistence in mouse tissue following topical LPP-10 siGLO treatment, wound beds received two doses. We removed the excess LPP-10 siRNA lying superficial to the healing tissue, prior to imaging. The LPPdelivered siGLO persisted for 5 days following the last application (Fig 4B-C). The results indicated that the LPP-10 vehicle effectively delivered siRNA to wound bed tissues and persisted for a duration adequate for therapeutic use.

3.4. LPP-10 siKeapl accelerates time to diabetic wound closure

To assess LPP efficacy in vivo, wounds on db/db mice were treated with LPP-10 siKeapl or siNS daily beginning 24 hours post-excision. The wounds were monitored until the re-epithelialized area resembled the surrounding intact skin (Fig 5A). LPP-10 siKeapl significantly accelerated time to closure to $22.25 \pm$ 1.97 days, when compared to $31.00 \pm 0.00$ days for LPP-10 siNS ( $\mathrm{p}=0.0213)$. The LPP-10 siKeapl 
treatment provided diabetic mice with healing times more similar to historical wild type C57/B6 mice (Fig 5B). Wound burden (pathologic healing - physiologic healing) in the LPP-10 siKeapl treated mice displayed a 57\% decrease relative to LPP-10 siNS treated mice (Fig 5C-D). Our data proved that siKeap1 delivery via LPP-10 significantly enhances the rate of diabetic wound healing and lessens wound burden.

\subsection{LPP-10 siKeapl augments regenerative wound environment}

Gene expression analysis performed on day 10 wound beds showed Keapl knockdown of $55.4 \pm 22.9 \%$ with daily LPP-10 siKeapl treatment, compared to LPP-10 siNS treated wounds ( $\mathrm{p}=0.004)$. There was no difference in liver Keapl mRNA levels (Fig 6A). This indicated that therapeutic effects of the LPP were localized and induced negligible systemic effects with topical application. We then explored differences in the mRNA expression of Nrf2 regulated antioxidant genes. The expression of antioxidant genes, $\mathrm{NAD}(\mathrm{P}) \mathrm{H}$ quinine dehydrogenase $1(N Q O-1)$, and manganese superoxide dismutase $(M n S O D)$ increased by $4.99 \pm 0.10$ fold $(\mathrm{p}=0.04)$ and $3.68 \pm 0.20$ fold $(\mathrm{p}=0.01)$, respectively, in LPP-10 siKeapl treated wounds compared to LPP-10 siNS treated wounds (Fig 6B). Additionally we explored the change in expression of various growth factors and chemokines related to wound healing and angiogenesis, in the intervention and control groups. The expression of the angiogenic chemokine, stromal cell-derived factor $1(S D F-1)$ increased $7.51 \pm 0.12$ fold $(\mathrm{p}=0.01)$ with Keapl silencing. Expression of epidermal growth factor $(E G F)$ increased $6.48 \pm 0.15$ fold $(\mathrm{p}=0.005)$ following Keapl silencing. Although the expressions of vascular endothelial growth factor (VEGF) and transforming growth factor alpha (TGF- $\alpha$ ) also increased following LPP-10 siKeapl treatment, they were not statistically significant (Fig 6C). Overall, the application of LPP-10 siRNA augmented expression of molecules involved in redox homeostasis and tissue regeneration.

Next, we analyzed the Keap1 and Nrf2 protein expression in 10 day wounds treated with LPP-10 siKeap1 and LPP-10 siNS. In the cytoplasmic fractions of the 10 day wound bed samples, Keap1 protein 
expression was decreased by $94.55 \pm 42.50 \%(\mathrm{p}=0.038)$ (Fig 6D), with LPP-10 siKeapl treatment. Following Keapl silencing, protein expression of Nrf2 in the corresponding nuclear fractions increased $3.27 \pm 1.63$ fold $(\mathrm{p}=0.039)$ compared to LPP-10 siNS controls (Fig 6E). These experiments showed that in vivo knockdown of Keapl via LPP-10 correlated with an increased protein expression of Nrf2, a critical transcription factor in maintenance of cell integrity.

To evaluate effects of Keapl knockdown on redox homeostasis and ROS handling in vivo, we used the luminol analog, L-012, and measured the resulting ROS-indicative bioluminescence in real time. The L012 substrate, which allows for the detection of $\mathrm{NAD}(\mathrm{P}) \mathrm{H}$ oxidase-derived ROS, was injected intraperitoneally into $\mathrm{db} / \mathrm{db}$ mice with 6-day-old wounds. Wounds treated daily with LPP-10 siKeapl had reduced bioluminescent signal compared to LPP-10 siNS treated wounds (Fig 6F). Quantification of bioluminescence from the wounds indicated significant reductions in ROS levels in LPP-10 siKeapl vs siNS treated wounds (43.97 \pm 1.93 vs $82.35 \pm 5.73$ bioluminescence counts, $\mathrm{p}<0.0001)$ (Fig 6G). With LPP-10 siKeapl treatment, the diabetic wounds demonstrated significant changes in redox homeostasis to correlate with the increased wound healing performance.

\subsection{LPP-10 siKeapl increases angiogenesis and granulation tissue in vivo}

In order to analyze whether gross observations and molecular analysis correspond to changes in wound architecture, we examined the quality of the tissue generated in 10 day old wounds treated with LPP-10 siKeap1. H\&E staining of wound tissue sections revealed that LPP-10 siKeapl treatment decreased the epithelial gap, the distance between the advancing edges of intact epithelium from the wound boundaries (Fig 7A). The epithelial gap significantly decreased from $8.07 \pm 1.37 \mathrm{~mm}$ in LPP-10 siNS treated wounds to $3.95 \pm 0.21 \mathrm{~mm}$ in LPP-10 siKeapl treated ones ( $\mathrm{p}=0.004)$ (Fig 7B). Additionally, we quantified the area of dermal granulation tissue, and this significantly increased from $1.15 \pm 0.44 \mathrm{~mm}^{2}$ in LPP-10 siNS treated wound to $2.15 \pm 0.65 \mathrm{~mm}^{2}$ in LPP-10 siKeapl treated wounds ( $\left.\mathrm{p}=0.049\right)$ (Fig 7 C-D). 
CD31, a marker for endothelial cells and neovascularization was increased in LPP-10 siKeapl treated mice. There was a mean of 109.83 \pm 17.96 CD31+ cells/hpf in LPP-10 siKeapl treated wounds and 71.16 $\pm 27.32 \mathrm{CD} 31+$ cells/hpf in LPP-10 siNS treated ones $(\mathrm{p}=0.02)($ Fig 7E-F). Additional histological analysis of completely re-epithelialized wounds showed that the underlying dermis of LPP-10 siKeapl treated wounds possessed more mature, organized collagen, similar to that in unwounded skin (Fig 7G). Wounds treated with LPP-10 siNS demonstrated sparse collagen deposition, corresponding to the deficiencies characteristic in diabetic wound healing. The healing data generated by treating diabetic mice wounds with novel LPP-10 siKeapl therapy demonstrates accelerated re-epithelialization and strongly correlates with the enhanced processes of neovascularization and granulation tissue formation.

\section{Discussion}

In this study we investigated the efficacy of a novel topical LPP siRNA delivery vehicle. We first explored the w/w/w ratio of DNC:CSP:siRNA to derive the ideal siRNA binding capacity, diameter, surface charge (particle stability), and siRNA load. Optimized ratios were then tested for cytotoxicity, ROS handling, in vitro transfection efficacy, and in vitro siRNA localization. We targeted the Keap1/Nrf2 pathway that has been previously been identified as a dysfunctional pathway playing a central role in the impaired diabetic wound healing phenotype. Transitioning to in vivo studies, we explored LPP-10 nanoparticle for penetration depth, siRNA persistence, transfection efficacy, downstream gene and protein expression changes, ROS management, and several measures of diabetic wound healing. Overall, we described the use of a novel LPP vehicle that includes a siRNA complexing protein, to successfully transport siRNA-based gene therapy to cutaneous diabetic murine wounds. Delivery of the siKeapl treatment produced several downstream effects related to redox homeostasis, neovascularization, and other areas of wound biology that significantly improved measures of wound healing. 
RNAi can be a very powerful tool to silence targeted genes, but its therapeutic use is limited by the availability of safe and effective delivery systems. For cutaneous diseases, topical or transdermal delivery are preferred over systemic delivery. Not only is it a direct route for targeting cells, it minimizes the risk of systemic off-target effects, and it is easy to apply. However, the stratum corneum is difficult to penetrate and endogenous RNAses degrade unprotected siRNAs. At the cellular level, both siRNA and cell membranes are anionic and tend to repel each other. To overcome these barriers, viral vectors and non-viral delivery vehicles have been developed, with lipids, peptides, and polymers among the most popular [29]. Peptide vehicles are notable for their safety and superior nucleotide condensation capabilities [30], and have been combined with lipid components to create hybrid delivery systems for nucleic acid transfection. This peptide-based route may prove useful for topical siRNA delivery, as more densely packaged siRNA will have an increased potential of reaching target cells with concentrations necessary to confer therapeutic efficacy. However, few such lipid-protein systems have been applied to topical siRNA delivery $[31,32]$. Additionally, others have found success with siRNAs that are covalently conjugated directly to proteins or gold nanoparticles, but the process of conjugation limits the ability to customize siRNA substitutions $[33,34]$. The relative ease of formulation and interchangeable parts in the LPP delivery vehicle allow for greater user-directed customizability. A wide range of siRNAs can be substituted to target different diseases with identified genetic components, conferring a distinct advantage to our nanoparticle.

Our novel LPP delivery system combines DNC, a CLN, with engineered CSP. Our previous work suggested CLN in a 6:1 w/w ratio of DNC successfully delivers siRNA [7]. We also found that liposomes with addition of CSP enhance transfection efficacy of plasmid DNA [10], highly suggesting that incorporation of CSP would boost siRNA delivery. Our CSP pentamer has been engineered to extensively exhibit positively charged surface residues in order to complex with negatively charged nucleic acids [10]. Therefore, we introduced CSP to the previous DNC (6:1) CLN in an effort to enhance siRNA condensation and improve topical siRNA delivery. CSP alone yields no mRNA knockdown (data not 
shown), suggesting that CSP likely only acts to enhance siRNA complexation with a CLN. This advantage in siRNA loading is suggested by the decrease in zeta potential upon adding siRNA-complexed CSP to nanoparticles with DNC (Fig 1E), possibly due to a coating effect and reduction in repulsive charges, where the DNC surface is draped in the protein/siRNA complex. The addition of increasing loads of siRNA slightly raises the zeta potential, as well as the diameter of LPP. These changes may be explained by greater surface charge of the DNC while the CSP interacts with siRNA. When the CSP is neutralized, it may slide into the hydrophobic region and contribute to maintaining the larger positive zeta potential due to the larger size. These changes suggest free siRNA molecules are encapsulated within the liposome and contribute to the swelling of the LPP. However, these changes in conformation with higher siRNA quantities do not have detrimental effects as the nanoparticle size is relatively unchanged, and the zeta potential remains above the well-described $30 \mathrm{mV}$ threshold for colloidal nanoparticle stability [27]. The elasticity of the DNC liposome component is also preserved upon complexing CSP and siRNA. Optimization of biophysical features and in vitro transfection efficacy led us to select the 10:1:1 DNC:CSP:siRNA formulation. The resulting LPP-10 with a minimally changed diameter and decreased surface charge may allow for more effective interaction between the DNC and cell membrane, to facilitate efficient siRNA delivery to target cells. Additional structural studies would shed light on the location of both CSP and siRNA in the overall complex. Pertinent to this study, the LPP-10 displayed consistent biophysical properties between batches (data not shown), and could easily be scaled up for mass production.

In our in vitro experiments, the optimal formulation was LPP-10, which successfully achieved $62 \%$ mRNA knockdown (Fig 3A). We observed efficient Keapl silencing in 3T3 cells using both gene and protein expression at multiple time points (Fig 3 A-E). Transfection efficacy using our LPP-10 nanoparticle was consistent with previous studies that successfully used charged peptides as nucleic acid condensing agents [10, 35-39]. While much smaller nanoparticles of 4-10 $\mathrm{nm}$ are able to penetrate the membrane bilayer directly, larger particles up to $120 \mathrm{~nm}$ in diameter rely on endocytosis, and even larger 
particles, such as LPP-10 (174 nm) may enter cells via micropinocytosis or phagocytosis [40]. Although the exact mechanism of cellular uptake, endosomal escape and release of siRNA from the LPP-10 delivery vehicle is unknown, it remains an interesting topic that requires further research. After applying LPP-10 carrying siKeapl we discovered expected upregulation of nuclear Nrf2 protein expression at 48 and 72-hour time points (Fig 3 D-E). This result served as proof of siRNA delivery and efficacy of LPP vehicle. While some liposomes have been reported to cause cytotoxicity [41, 42], our LPP in various formulation ratios showed no apparent toxicity in vitro, which is ideal for application to diabetic wounds (Fig 3F). These preliminary measures of efficacy and safety supported the transition of the LPP-10 vehicle into animal models.

Using a murine diabetic wound healing model, we established that by 10 days of daily LPP-10 siKeapl treatment, the siRNA penetrated deep into the target tissue (Fig 4A). Additionally, we found that the LPP10-delivered siRNA persisted in mouse skin for 5 days after most recent application (Fig 4B-C), which could help simplify treatment regimens and limit cost to patients. Further murine studies revealed that LPP-10 siKeapl locally silenced Keapl and allowed Nrf2 to be upregulated, while expression levels of Keapl at distant sites such as the liver were unaffected (Fig 6A-B). This was consistent with Keapl knockdown using Lipofectamine method previously used by our group [20]. However, the transfection agent used (Lipofectamine) was cited for toxicity concerns [43, 44]. For this reason, LPP was developed as our next generation delivery vehicle. LPP-10 siKeapl therapy resulted in significantly accelerated wound closure (Fig 5A) and diminished wound burden (Fig 5 B, D). Corresponding to this result, we observed a decreased epithelial gap and increased granulation tissue formation with CD31+ cells in 10 day wounds following daily treatment with LPP-10 siKeapl (Fig 7 A-F).

Based on the central role of Nrf2 in redox homeostasis, we measured levels of ROS and found that LPP10 siKeap1 therapy resulted in a significant decrease in oxidative stress in mouse diabetic wound beds (Fig 6F-G). This result was reflective of in vitro assessment of intracellular ROS in fibroblasts, following 
transfection with LPP-10 siKeap1, and reinforcing the transfection efficiency of our delivery vehicle (Fig 3). These results further corroborate our recently reported observations of altering Keap1/Nrf2 pathways in diabetic wound healing [20]. In the present study, we also discovered that gene expression of Nrf2mediated redox related enzymes, NQO-1 and MnSOD, were upregulated (Fig 6B). Both NQO-1 and MnSOD are critical antioxidants in redox homeostasis, and contributed to the observed reduction in ROS in LPP-10 siKeapl treated wounds.

In addition to upregulating antioxidant enzymes, we discovered that $\mathrm{Nrf} 2$ is possibly associated with other mechanisms that serve to accelerate wound healing. To further explore the molecular environment of the wound bed following Nrf2 activation by LPP-10 siKeapl, we measured gene expression of chemokines and growth factors related to wound healing. Interestingly, $S D F-1$ and $E G F$ were significantly upregulated following Keapl silencing but VEGF and TGF- $\alpha$ were not. While SDF-1 has been associated with vascular repair and remodeling in wound healing $[18,45]$, there is a lack of literature describing the relationship of SDF-1 and Nrf2. Our results are supported by Yang et al. who revealed that both SDF-1 and Nrf2 could be regulated through interactions with inflammatory molecules such as IL-1 $\alpha$ [46].

Given the significant health burden posed by poor diabetic wound healing, the development of a topical treatment that promotes cutaneous wound healing would greatly benefit diabetic patients with non-healing wounds. As our understanding of the pathology of wound healing in diabetes develops, the number of therapeutic targets expands $[13,47]$. Successful gene therapies for diabetic wound healing in mice include siRNA-mediated ganglioside GM3 synthase knockdown to increase keratinocyte migration and proliferation [48], plasmid VEGF delivery via hyaluronic acid-matrix metalloproteinase hydrogels [49], siRNA mediated xanthine dehydrogenase knockdown to decrease overproduction of ROS [50], siRNA mediated prolyl-hydroxylase domain 2 knockdown to increase angiogenic factors and fibroblast proliferation [51, 52], and siRNA knockdown of p53 to augment vasculogenic mediators [22]. Many of 
these approaches showed efficacy but are limited by narrow targets that do not have the broad downstream effects that the transcription factor Nrf2 induces.

Refinement of the LPP delivery system will further develop our promising candidate for clinical topical siRNA delivery. As it is currently formulated, the LPP shelf life may require modifications to ensure structural and functional longevity of the nanoparticle. Incorporation of stabilizers such as PEGylated moieties may prolong usage timeframe, but may also increase systemic bioavailability and increase offtarget effects [53]. Additional investigation into the mechanism of cellular uptake, endosomal escape, cytosolic localization, and intracellular siRNA release will provide insights into optimizing the design of the nanoparticle. Possible modifications may include incorporating the LPP into a gel that can hydrate the epidermis and facilitate nanoparticle penetration to the dermis and subcutaneous tissues. Future studies may explore details of distribution of the LPP, siRNA release, and persistence once within skin tissue. Another exciting component of our novel LPP is the central pore within CSP, which can encapsulate a small drug molecular. Addition of a drug with siRNA may further enhance treatment efficacy through simultaneously targeting multiple pathways [12]. Synergistic therapies have proven useful in the treatment of many diseases including diabetic wounds [54-56], and LPP offers this exciting potential in a safe and effective manner.

\section{Conclusions}

Our novel LPP currently stands as a viable and efficient topical siRNA delivery system for the treatment of diabetic wounds. Its ability to package and deliver treatment in a safe, efficient, and localized manner are key findings to support further trials and to translate this technology for clinical use in all cutaneous diseases with a significant identified genetic component. 


\section{Acknowledgements}

This work was supported by the American Diabetes Association "Pathway to Stop Diabetes" to D.C. [grant number 1-16-ACE-08], the NYU Applied Research Support Fund to P.R., and the National Science Foundation to J.K.M. [grant numbers DMR-1505214, IIP-144983]. We are grateful to the Histopathology Core, Immunohistochemistry Core, and Microscopy Core at NYU Langone Medical Center, supported in part by grant UL1 TR00038 from the National Center for Advancing Translational Sciences (NCATS), National Institutes of Health, in part by the Laura and Isaac Perlmutter Cancer Center Support Grant; NIH /NCI P30CA016087 and the National Institutes of Health S10 Grants; NIH/ORIP S10OD01058 and S10OD018338.

\section{Author Contributions}

PSR, AZ, ZMB designed and performed experiments, analyzed data and prepared manuscript, JAF performed experiments, analyzed data and reviewed manuscript, NS, HTM, WJR, JAD, SJB, RC, SH, MHJ, CK, RAS performed experiments, PBS reviewed manuscript, JKM designed constructs, some experiments and reviewed manuscript, DJC designed experiments and reviewed manuscript. 


\section{References}

[1] Centers for Disease Control and Prevention. National Diabetes Statistics Report: Estimates of Diabetes and Its Burden in the United States, US Department of Health and Human Services, Atlanta, GA, 2014.

[2] C. Dunnill, T. Patton, J. Brennan, J. Barrett, M. Dryden, J. Cooke, D. Leaper, N.T. Georgopoulos, Reactive oxygen species (ROS) and wound healing: the functional role of ROS and emerging ROSmodulating technologies for augmentation of the healing process, Int Wound J 14(1) (2017) 89-96. [3] G.L. Sen, H.M. Blau, A brief history of RNAi: the silence of the genes, FASEB J 20(9) (2006) 12939.

[4] J. Zhou, K.T. Shum, J.C. Burnett, J.J. Rossi, Nanoparticle-Based Delivery of RNAi Therapeutics: Progress and Challenges, Pharmaceuticals (Basel) 6(1) (2013) 85-107.

[5] S. Bracke, M. Carretero, S. Guerrero-Aspizua, E. Desmet, N. Illera, M. Navarro, J. Lambert, M. Del Rio, Targeted silencing of DEFB4 in a bioengineered skin-humanized mouse model for psoriasis: development of siRNA SECosome-based novel therapies, Exp Dermatol 23(3) (2014) 199-201.

[6] B. Geusens, M.V. Gele, S. BRaat, S.D. Smedt, M. Stuart, T. Prow, Flexible nanosomes (SECosomes) enable efficient siRNA delivery in cultured primary skin cells and in the viable epidermis of ex vivo human skin, Adv. Funct. Mater 20(23) (2010) 4077-4090.

[7] M. Ham, M. Soares, A. Zhou, M. Ayaz, M. Wetterau, P. Lofti, F. Lalezarzadeh, S. WIlson, C. Ojo, A. Bandekar, S. Sofou, D. Ceradini, P. Saadeh, Abstract 123: Topical gene silencing towards an off-theshelf solution, Plast. Reconstr. Surg. 131(5S) (2013) 93.

[8] M. Chen, M. Zakrewsky, V. Gupta, A.C. Anselmo, D.H. Slee, J.A. Muraski, S. Mitragotri, Topical delivery of siRNA into skin using SPACE-peptide carriers, Journal of controlled release : official journal of the Controlled Release Society 179 (2014) 33-41.

[9] H. Hatakeyama, E. Ito, H. Akita, M. Oishi, Y. Nagasaki, S. Futaki, H. Harashima, A pH-sensitive fusogenic peptide facilitates endosomal escape and greatly enhances the gene silencing of siRNA- 
containing nanoparticles in vitro and in vivo, Journal of controlled release : official journal of the Controlled Release Society 139(2) (2009) 127-32.

[10] H.T. More, J.A. Frezzo, J. Dai, S. Yamano, J.K. Montclare, Gene delivery from supercharged coiled-coil protein and cationic lipid hybrid complex, Biomaterials 35(25) (2014) 7188-93.

[11] B.R. McNaughton, J.J. Cronican, D.B. Thompson, D.R. Liu, Mammalian cell penetration, siRNA transfection, and DNA transfection by supercharged proteins, Proceedings of the National Academy of Sciences 106(15) (2009) 6111-6116.

[12] S.K. Gunasekar, M. Asnani, C. Limbad, J.S. Haghpanah, W. Hom, H. Barra, S. Nanda, M. Lu, J.K. Montclare, N-terminal aliphatic residues dictate the structure, stability, assembly, and small molecule binding of the coiled-coil region of cartilage oligomeric matrix protein, Biochemistry 48(36) (2009) 8559-8567.

[13] H. Brem, M. Tomic-Canic, Cellular and molecular basis of wound healing in diabetes, J Clin Invest 117(5) (2007) 1219-22.

[14] C.L. Hess, M.A. Howard, C.E. Attinger, A review of mechanical adjuncts in wound healing: hydrotherapy, ultrasound, negative pressure therapy, hyperbaric oxygen, and electrostimulation, Annals of plastic surgery 51(2) (2003) 210-8.

[15] L. Yazdanpanah, M. Nasiri, S. Adarvishi, Literature review on the management of diabetic foot ulcer, World J Diabetes 6(1) (2015) 37-53.

[16] C.C. Lan, C.S. Wu, S.M. Huang, I.H. Wu, G.S. Chen, High-glucose environment enhanced oxidative stress and increased interleukin-8 secretion from keratinocytes: new insights into impaired diabetic wound healing, Diabetes 62(7) (2013) 2530-8.

[17] A.G. Ojalvo, J.B. Acosta, Y.M. Mari, M.F. Mayola, C.V. Perez, W.S. Gutierrez, Marichal, II, E.A. Seijas, A.M. Kautzman, A.E. Pacheco, D.G. Armstrong, Healing enhancement of diabetic wounds by locally infiltrated epidermal growth factor is associated with systemic oxidative stress reduction, Int Wound J 14(1) (2017) 214-225. 
[18] D.J. Ceradini, D. Yao, R.H. Grogan, M.J. Callaghan, D. Edelstein, M. Brownlee, G.C. Gurtner, Decreasing intracellular superoxide corrects defective ischemia-induced new vessel formation in diabetic mice, J Biol Chem 283(16) (2008) 10930-8.

[19] J.A. Nogueira-Machado, F.C. Lima e Silva, L.O. Medina, D.C. Costa, M.M. Chaves, Modulation of the reactive oxygen species (ROS) generation mediated by cyclic AMP-elevating agents or Interleukin 10 in granulocytes from type 2 diabetic patients (NIDDM): a PKA-independent phenomenon, Diabetes Metab 29(5) (2003) 533-7.

[20] M.A. Soares, O.D. Cohen, Y.C. Low, R.A. Sartor, T. Ellison, U. Anil, L. Anzai, J.B. Chang, P.B. Saadeh, P.S. Rabbani, D.J. Ceradini, Restoration of Nrf2 Signaling Normalizes the Regenerative Niche, Diabetes 65(3) (2016) 633-46.

[21] L. Baird, A.T. Dinkova-Kostova, The cytoprotective role of the Keap1-Nrf2 pathway, Arch Toxicol 85(4) (2011) 241-72.

[22] P.D. Nguyen, J.P. Tutela, V.D. Thanik, D. Knobel, R.J. Allen, Jr., C.C. Chang, J.P. Levine, S.M. Warren, P.B. Saadeh, Improved diabetic wound healing through topical silencing of p53 is associated with augmented vasculogenic mediators, Wound Repair Regen 18(6) (2010) 553-9.

[23] V.D. Thanik, M.R. Greives, O.Z. Lerman, N. Seiser, W. Dec, C.C. Chang, S.M. Warren, J.P. Levine, P.B. Saadeh, Topical matrix-based siRNA silences local gene expression in a murine wound model, Gene Ther 14(17) (2007) 1305-8.

[24] R. Hunter, Colloid Science, Zeta Potential in Colloid Science Academic Press (1981) 2.

[25] C. Rota, C.F. Chignell, R.P. Mason, Evidence for free radical formation during the oxidation of 2'7'-dichlorofluorescin to the fluorescent dye 2'-7'-dichlorofluorescein by horseradish peroxidase: possible implications for oxidative stress measurements, Free Radic. Biol. Med. 27(7-8) (1999) 87381. 
[26] R.D. Galiano, J. Michaels, M. Dobryansky, J.P. Levine, G.C. Gurtner, Quantitative and reproducible murine model of excisional wound healing, Wound Repair Regen 12(4) (2004) 48592.

[27] R.H. Muller, C. Jacobs, O. Kayser, Nanosuspensions as particulate drug formulations in therapy. Rationale for development and what we can expect for the future, Adv Drug Deliv Rev 47(1) (2001) 3-19.

[28] D.R. D'Souza, M.M. Salib, J. Bennett, M. Mochin-Peters, K. Asrani, S.E. Goldblum, K.J. Renoud, P. Shapiro, A. Passaniti, Hyperglycemia regulates RUNX2 activation and cellular wound healing through the aldose reductase polyol pathway, J Biol Chem 284(27) (2009) 17947-55.

[29] T. Musacchio, V.P. Torchilin, siRNA delivery: from basics to therapeutic applications, Front Biosci (Landmark Ed) 18 (2013) 58-79.

[30] J. Beloor, S. Zeller, C.S. Choi, S.K. Lee, P. Kumar, Cationic cell-penetrating peptides as vehicles for siRNA delivery, Ther Deliv 6(4) (2015) 491-507.

[31] A.D. Tagalakis, S. Castellaro, H. Zhou, A. Bienemann, M.M. Munye, D. McCarthy, E.A. White, S.L. Hart, A method for concentrating lipid peptide DNA and siRNA nanocomplexes that retains their structure and transfection efficiency, International journal of nanomedicine 10 (2015) 2673-83.

[32] J.A. Zuris, D.B. Thompson, Y. Shu, J.P. Guilinger, J.L. Bessen, J.H. Hu, M.L. Maeder, J.K. Joung, Z.Y. Chen, D.R. Liu, Cationic lipid-mediated delivery of proteins enables efficient protein-based genome editing in vitro and in vivo, Nat Biotechnol 33(1) (2015) 73-80.

[33] X. Yi, G. Zhao, H. Zhang, D. Guan, R. Meng, Y. Zhang, Q. Yang, H. Jia, K. Dou, C. Liu, F. Que, J.Q. Yin, MITF-siRNA formulation is a safe and effective therapy for human melasma, Mol Ther 19(2) (2011) $362-71$.

[34] D. Zheng, D.A. Giljohann, D.L. Chen, M.D. Massich, X.Q. Wang, H. Iordanov, C.A. Mirkin, A.S. Paller, Topical delivery of siRNA-based spherical nucleic acid nanoparticle conjugates for gene regulation, Proc Natl Acad Sci U S A 109(30) (2012) 11975-80. 
[35] J.L. Chen, H. Wang, J.Q. Gao, H.L. Chen, W.Q. Liang, Liposomes modified with polycation used for gene delivery: preparation, characterization and transfection in vitro, International journal of pharmaceutics 343(1-2) (2007) 255-61.

[36] X. Gao, L. Huang, Potentiation of cationic liposome-mediated gene delivery by polycations, Biochemistry 35(3) (1996) 1027-36.

[37] S. Li, L. Huang, In vivo gene transfer via intravenous administration of cationic lipid-protamineDNA (LPD) complexes, Gene Ther 4(9) (1997) 891-900.

[38] J. Schäfer, S. Höbel, U. Bakowsky, A. Aigner, Liposome-polyethylenimine complexes for enhanced DNA and siRNA delivery, Biomaterials 31(26) (2010) 6892-900.

[39] S. Yamano, J. Dai, S. Hanatani, K. Haku, T. Yamanaka, M. Ishioka, T. Takayama, C. Yuvienco, S. Khapli, A.M. Moursi, J.K. Montclare, Long-term efficient gene delivery using polyethylenimine with modified Tat peptide, Biomaterials 35(5) (2014) 1705-15.

[40] Z. Mao, X. Zhou, C. Gao, Influence of structure and properties of colloidal biomaterials on cellular uptake and cell functions, Biomater. Sci 1 (2013) 896-911.

[41] K. Romøren, B.J. Thu, N.C. Bols, Ø. Evensen, Transfection efficiency and cytotoxicity of cationic liposomes in salmonid cell lines of hepatocyte and macrophage origin, Biochimica et Biophysica Acta (BBA) - Biomembranes 1663(1-2) (2004) 127-134.

[42] K. Lappalainen, I. Jääskeläinen, K. Syrjänen, A. Urtti, S. Syrjänen, Comparison of Cell Proliferation and Toxicity Assays Using Two Cationic Liposomes, Pharmaceutical Research 11(8) (1994) 1127-1131.

[43] K.D. Mack, R. Wei, A. Elbagarri, N. Abbey, M.S. McGrath, A novel method for DEAE-dextran mediated transfection of adherent primary cultured human macrophages, J Immunol Methods 211(1-2) (1998) 79-86. 
[44] Y.Q. Zhong, J. Wei, Y.R. Fu, J. Shao, Y.W. Liang, Y.H. Lin, J. Liu, Z.H. Zhu, [Toxicity of cationic liposome Lipofectamine 2000 in human pancreatic cancer Capan-2 cells], Nan Fang Yi Ke Da Xue Xue Bao 28(11) (2008) 1981-4.

[45] E.R. Fedyk, D. Jones, H.O. Critchley, R.P. Phipps, T.M. Blieden, T.A. Springer, Expression of stromal-derived factor-1 is decreased by IL-1 and TNF and in dermal wound healing, J Immunol 166(9) (2001) 5749-54.

[46] B. Yang, W. Li, Q. Zheng, T. Qin, K. Wang, J. Li, B. Guo, Q. Yu, Y. Wu, Y. Gao, X. Cheng, S. Hu, S.N. Kumar, S. Liu, Z. Song, Transforming growth factor beta-activated kinase 1 negatively regulates interleukin-1alpha-induced stromal-derived factor-1 expression in vascular smooth muscle cells, Biochem Biophys Res Commun 463(1-2) (2015) 130-6.

[47] E. Tsourdi, A. Barthel, H. Rietzsch, A. Reichel, S.R. Bornstein, Current aspects in the pathophysiology and treatment of chronic wounds in diabetes mellitus, Biomed Res Int 2013 (2013) 385641.

[48] P.S. Randeria, M.A. Seeger, X.Q. Wang, H. Wilson, D. Shipp, C.A. Mirkin, A.S. Paller, siRNA-based spherical nucleic acids reverse impaired wound healing in diabetic mice by ganglioside GM3 synthase knockdown, Proc Natl Acad Sci U S A 112(18) (2015) 5573-8.

[49] T. Tokatlian, C. Cam, T. Segura, Porous hyaluronic acid hydrogels for localized nonviral DNA delivery in a diabetic wound healing model, Advanced healthcare materials 4(7) (2015) 1084-91.

[50] A.L. Weinstein, F.D. Lalezarzadeh, M.A. Soares, P.B. Saadeh, D.J. Ceradini, Normalizing dysfunctional purine metabolism accelerates diabetic wound healing, Wound Repair Regen 23(1) (2015) 14-21.

[51] M. Wetterau, F. George, A. Weinstein, P.D. Nguyen, J.P. Tutela, D. Knobel, O. Cohen Ba, S.M. Warren, P.B. Saadeh, Topical prolyl hydroxylase domain-2 silencing improves diabetic murine wound closure, Wound Repair Regen 19(4) (2011) 481-6. 
[52] X. Zhang, X. Yan, L. Cheng, J. Dai, C. Wang, P. Han, Y. Chai, Wound healing improvement with PHD-2 silenced fibroblasts in diabetic mice, PLoS ONE 8(12) (2013) e84548.

[53] M.L. Immordino, F. Dosio, L. Cattel, Stealth liposomes: review of the basic science, rationale, and clinical applications, existing and potential, International journal of nanomedicine 1(3) (2006) 297.

[54] R.J. Allen, M.A. Soares, I.D. Haberman, C. Szpalski, J. Schachar, C.D. Lin, P.D. Nguyen, P.B. Saadeh, S.M. Warren, Combination therapy accelerates diabetic wound closure, PLoS ONE 9(3) (2014) e92667.

[55] L. Scherer, J.J. Rossi, M.S. Weinberg, Progress and prospects: RNA-based therapies for treatment of HIV infection, Gene Ther 14(14) (2007) 1057-64.

[56] M. Vanneman, G. Dranoff, Combining immunotherapy and targeted therapies in cancer treatment, Nat Rev Cancer 12(4) (2012) 237-51. 


\section{Figure Captions}

Figure 1. LPP complexes with siRNA to form stable nanoparticle. Electrophoretic mobility shift assay of increasing w/w ratios of DNC with siRNA, siRNA weight is constant for all samples (A), and increasing w/w ratios of CSP with siRNA, siRNA weight is constant for all samples (B). Electrophoretic mobility shift assay of increasing w/w/w ratios of DNC:CSP:siRNA, CSP and siRNA weights are constant across samples (C). LPP diameter measurements (D) and surface

charge zeta potential measurements (E) of w/w/w DNC:CSP:siRNA ratios, with increasing weights of CSP, and constant weights of DNC and siRNA. LPP diameter (F) and zeta potential measurements $(\mathrm{G})$ with increasing amounts of siRNA load.

Figure 2. Liposome diameter decreases upon CSP incorporation. TEM of DNC:CSP:siRNA in the ratios 10:0:0 (A), 10:0:1 (B), and 10:1:1 (C). Dynamic light scattering and diameter measurements from TEM (D). Scale bar, 100nm.

Figure 3. LPP is an efficient and safe in vitro siRNA delivery vehicle. In vitro GAPDH gene knockdown analysis in 3T3 fibroblasts transfected with lipofectamine, lipoplex, and varying ratios of LPP siGAPDH (A). Analysis of gene knockdown specificity in 3T3 cells, after transfection with 10:1:1 LPP siNonsense (siNS) and 10:1:1 siGAPDH (B). In vitro Keapl gene expression analysis at 24, 48, and 72 hours after transfection with 10:1:1 LPP siKeapl (C). Protein expression in cytoplasmic fraction of 3T3 cells transfected with 10:1:1 LPP siKeapl at indicated times (D). Protein expression in nuclear fraction of 3T3 cells transfected with 10:1:1 LPP siKeapl at indicated times (E). Flow cytometric evaluation of intracellular ROS in cells, transfected with reagents as indicated, using $\mathrm{CM}-\mathrm{H}_{2} \mathrm{DCFDA}(\mathrm{F})$. In vitro cytotoxicity assay following transfection with lipofectamine and various LPP ratios $(\mathrm{G})$. Photograph of cells transfected in vitro with 
DNC:siGLO (H i) and DNC:CSP:siGLO (Hii).siGLO fluoresces red, nuclei are stained with DAPI.

Figure 4. LPP delivered siRNA reaches subcutaneous tissue and persists for several days. Photographs of wound bed tissue sections at 3, 7 and 10 days following excision and application of LPP-10 siGLO (A) ( $\triangleright=$ =epithelium; $\$$ =dermis; $\odot=$ =adipose tissue; $\square=$ panniculus carnosus muscle). Clearance of LPP-10 siGLO from diabetic mouse wounds. In vivo imaging at 0 (removal of remnant LPP-10 siGLO), 3, 5 and 7 days, following 2 days of LPP-10 siGLO application (B). Quantification of siGLO fluorescence intensity in B (C). Sections are counterstained with DAPI.

Figure 5. Topical LPP-10 siKeapl gene therapy accelerates diabetic wound closure. Photographs of stented wounds on diabetic mice with daily treatments as indicated (A). Mean time to closure of wounds (B). Percentage non-healed wound area per treatment (C). Wound burden (pathologic healing - physiologic healing) per treatment (D).

Figure 6. Topical LPP-10 siKeapl alters diabetic wound environment. Keapl gene expression in 10 day diabetic wounds and liver, following topical LPP-10 siKeapl therapy (A). Gene expression of antioxidants (B) and growth factors (C) in wound beds, after 10 days of treatment with LPP-10 siKeap1. Western blot and quantification of Keap1 protein in cytoplasmic lysates of 10d treated wounds (D). Western blot and quantification of Nrf2 from nuclear lysates of 10 day treated wounds. (E). Real-time in vivo ROS measurement in 6d treated wounds using L-012 (F). Quantification of L-012 luminescence in F (G).

Figure 7. Topical siKeapl gene therapy improves the histologic profile of diabetic wounds. H\&E-stained sections demonstrate epithelial gap of 10d wounds treated with LPP-10 siNonsense or siKeap1 
(A). Quantification of the epithelial gap (B). Granulation tissue in $10 \mathrm{~d}$ wound beds treated with LPP-10 as indicated (The yellow dashed-line indicates granulation tissue and wounds are oriented to the right of photograph) (C). Quantification of granulation tissue (D). CD31 immunohistochemistry on 10d wound tissue sections, treated with LPP-10 as indicated (E). Quantification of CD31+ cells per high power field in 10d LPP-treated granulation tissue (F). Histological examination of healed wounds and collagen deposition $(\mathrm{G})$. 
$1 \mathrm{~A}$

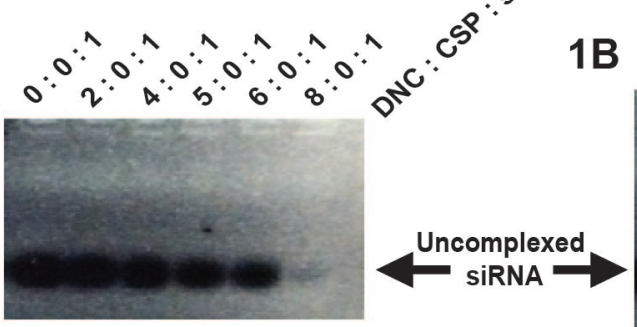

$1 \mathrm{C}$

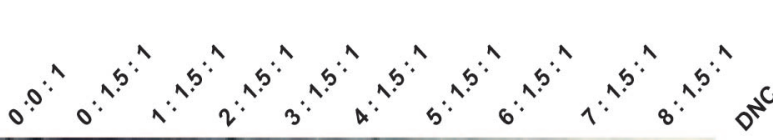

1D

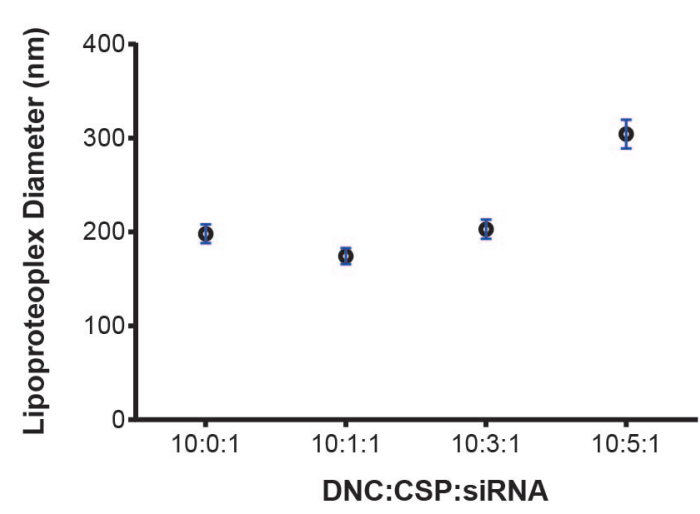

$1 F$

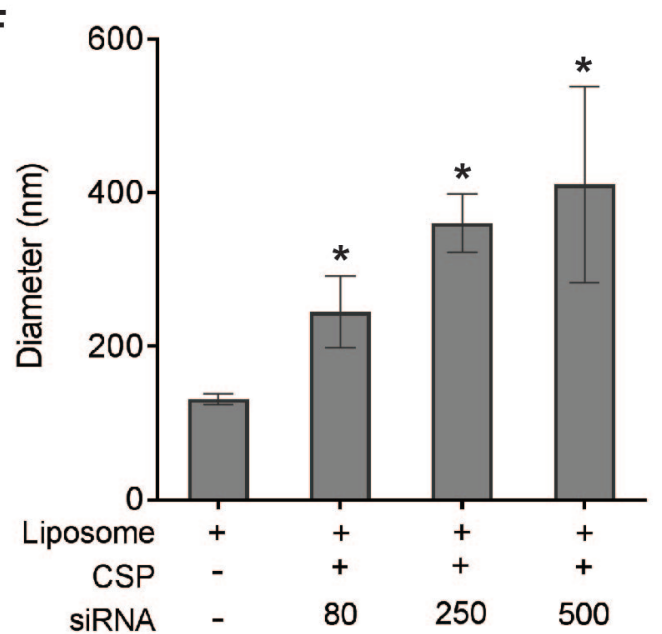

$1 E$
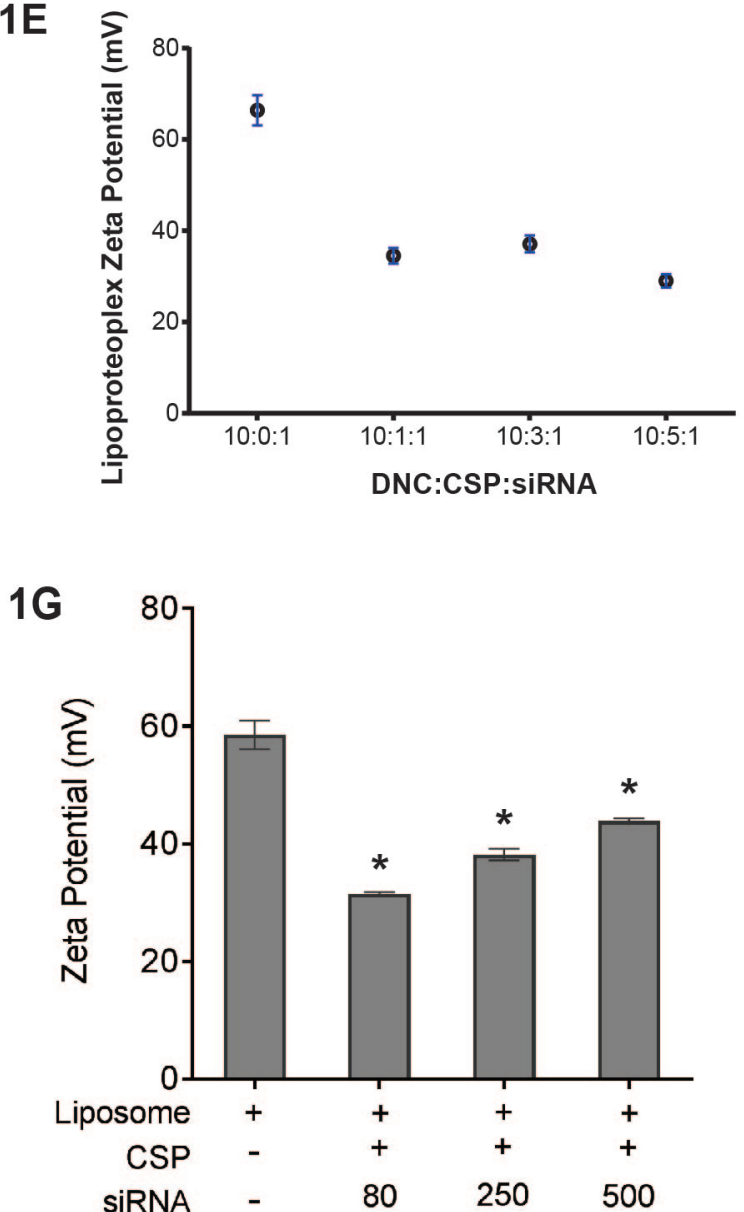


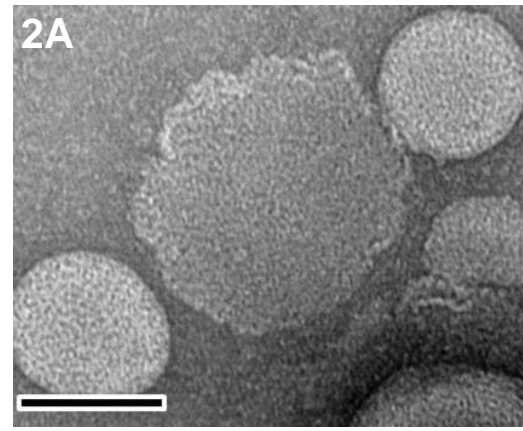

DNC:CSP:siRNA (10:0:0)

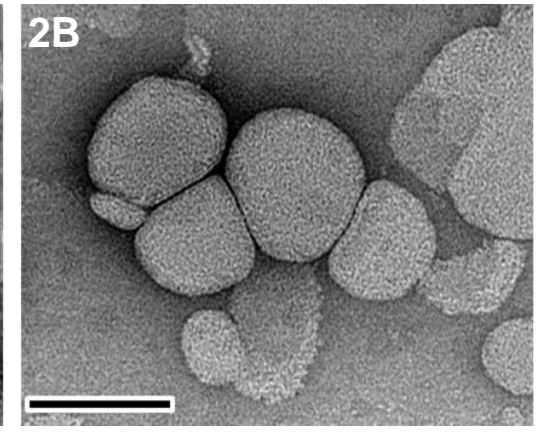

DNC:CSP:siRNA (10:0:1)

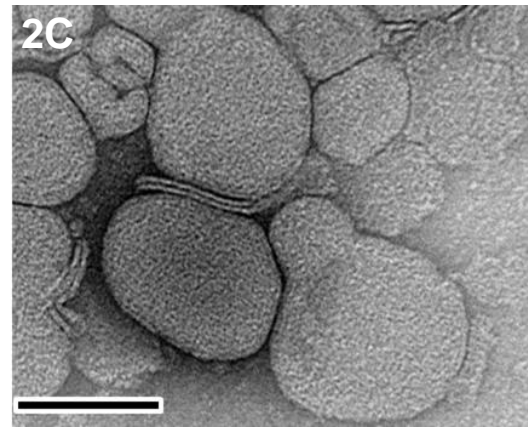

DNC:CSP:siRNA (10:1:1)

2D

\begin{tabular}{|l|l|l|}
\hline Samples & Dynamic Light Scattering $(\mathbf{n m})$ & TEM $(\mathbf{n m})$ \\
\hline DNC:CSP:siRNA (10:0:0) & $131.11 \pm 7.33$ & $126.64 \pm 14.67$ \\
\hline DNC:CSP:siRNA (10:0:1) & $198.08 \pm 9.09$ & $176.70 \pm 47.83$ \\
\hline DNC:CSP:siRNA (10:1:1) & $174.22 \pm 8.71$ & $160.94 \pm 34.21$ \\
\hline
\end{tabular}




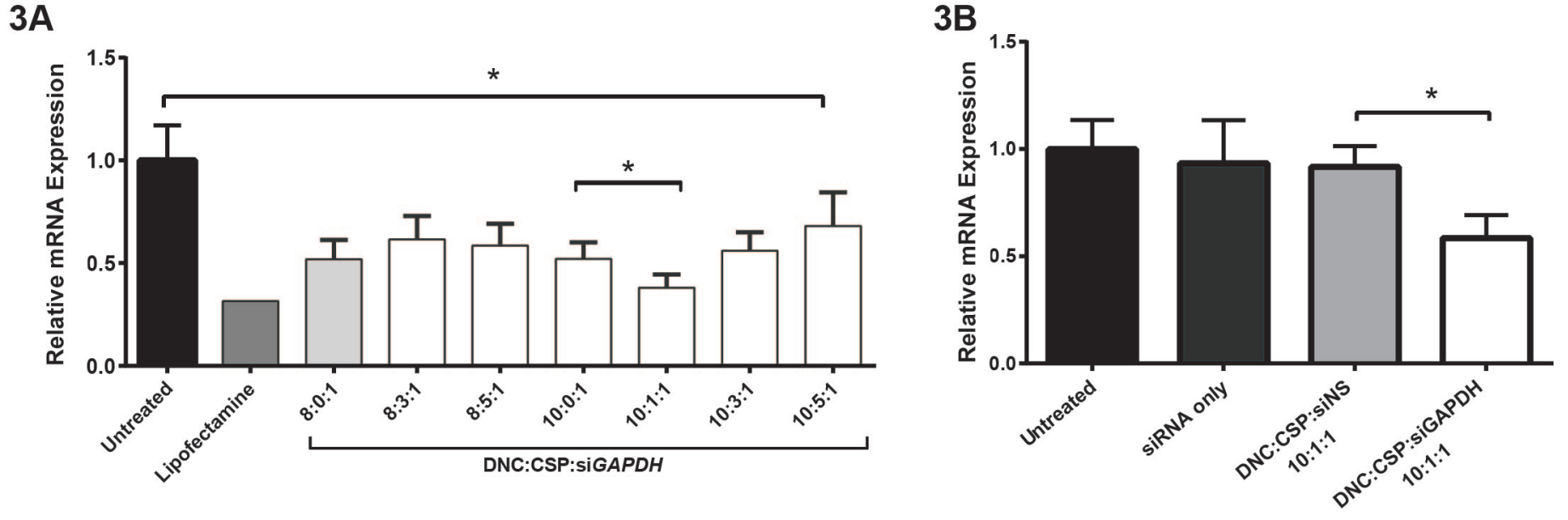

$3 C$
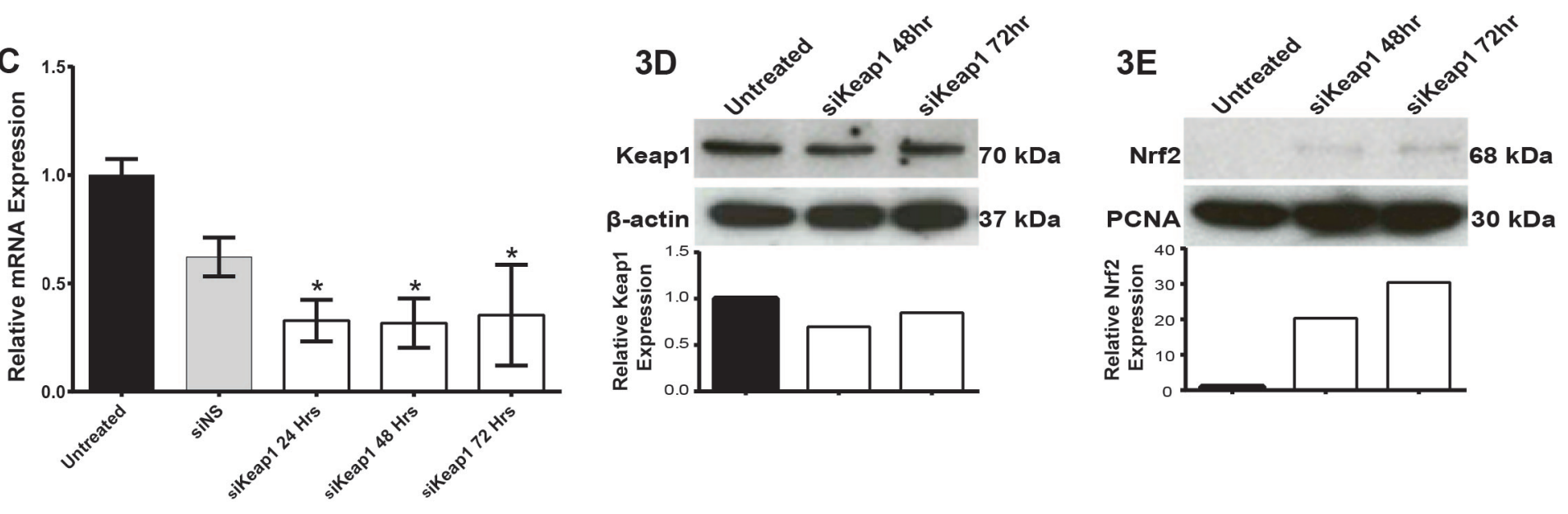

$3 F$

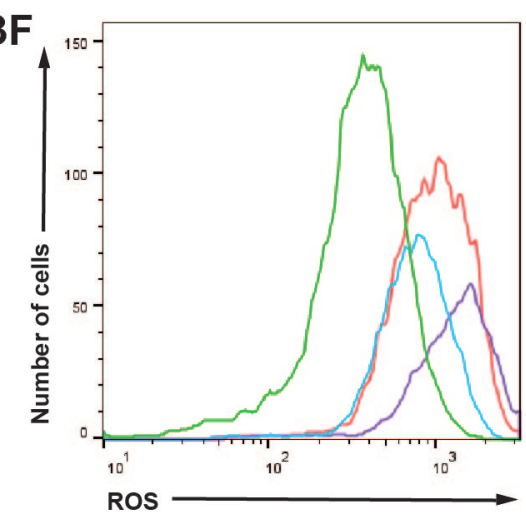

10:1:1 DNC:CSP:siKeap1

10:1:1 DNC:CSP:siNS

10:0:1 DNC:CSP:siKeap1
10:0:1 DNC:CSP:siNS
$3 G$

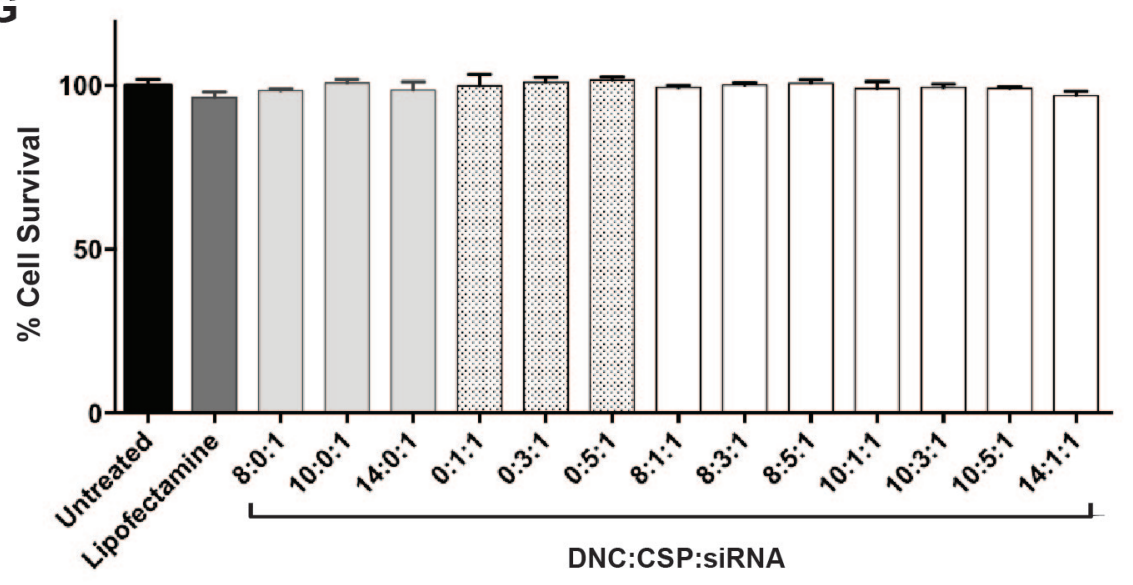

$3 \mathrm{H}$
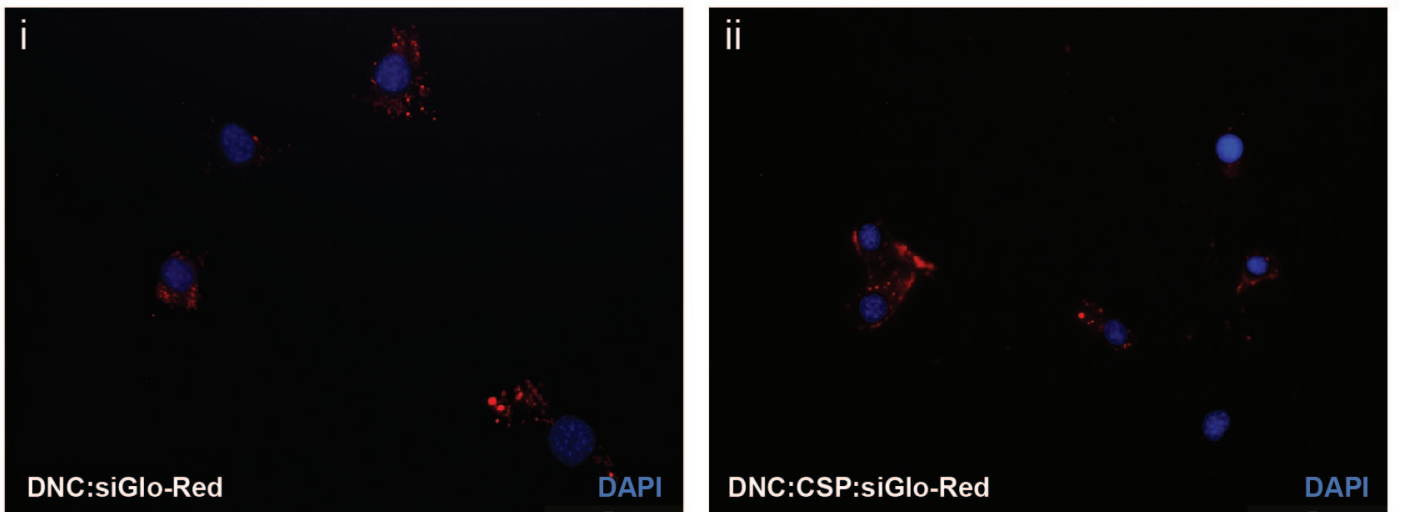

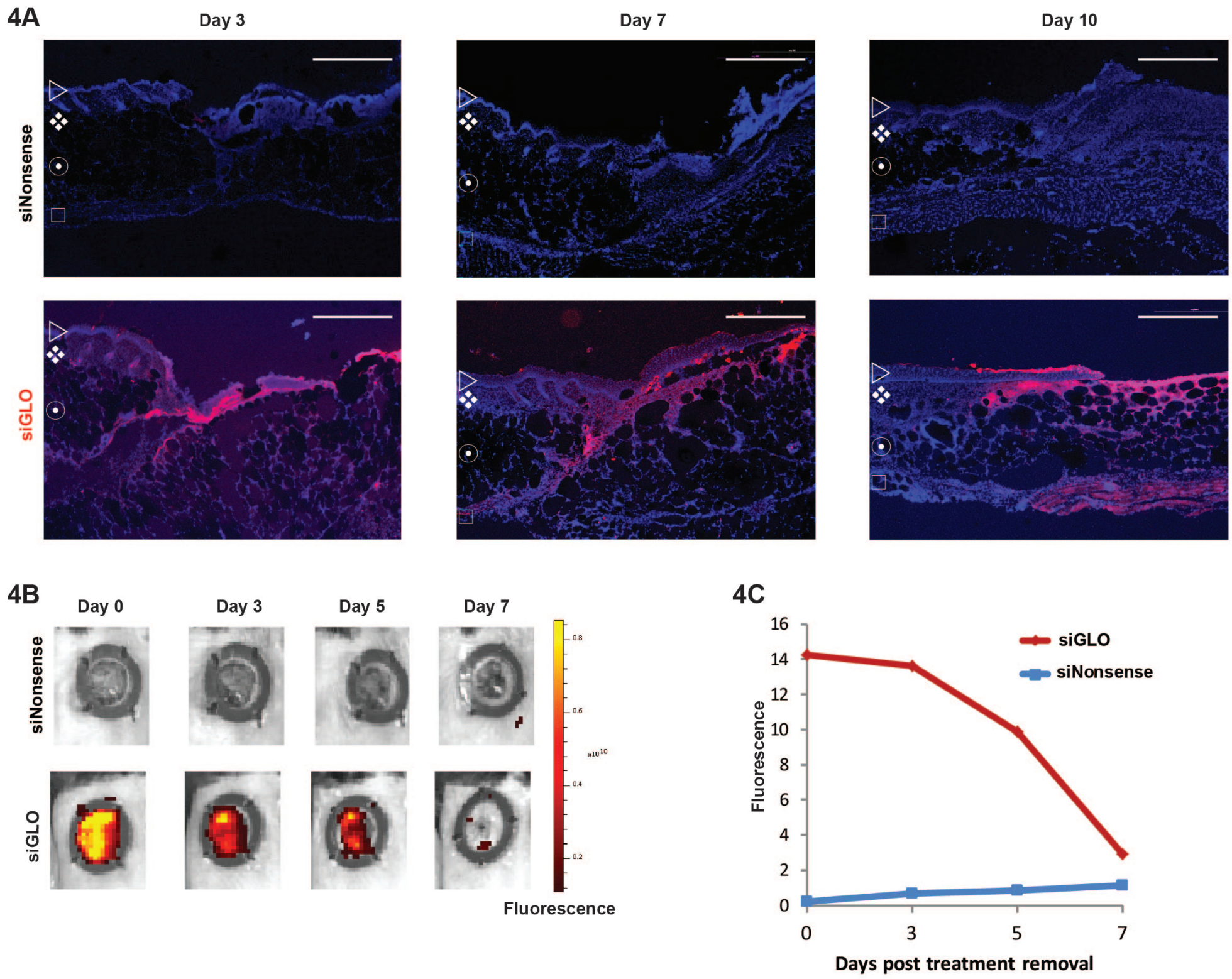


\section{$5 \mathrm{~A}$}
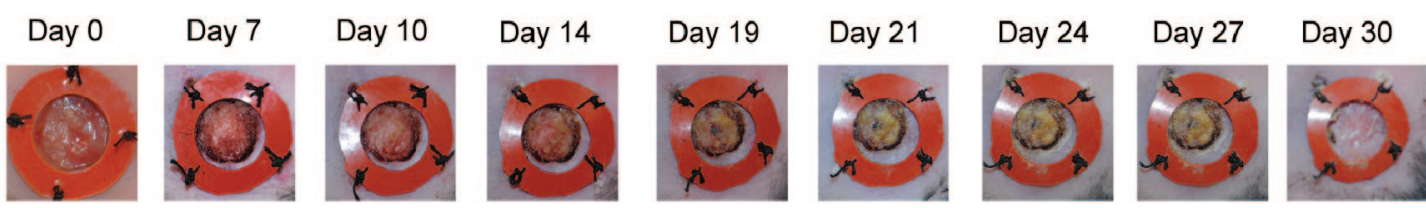

Day 32
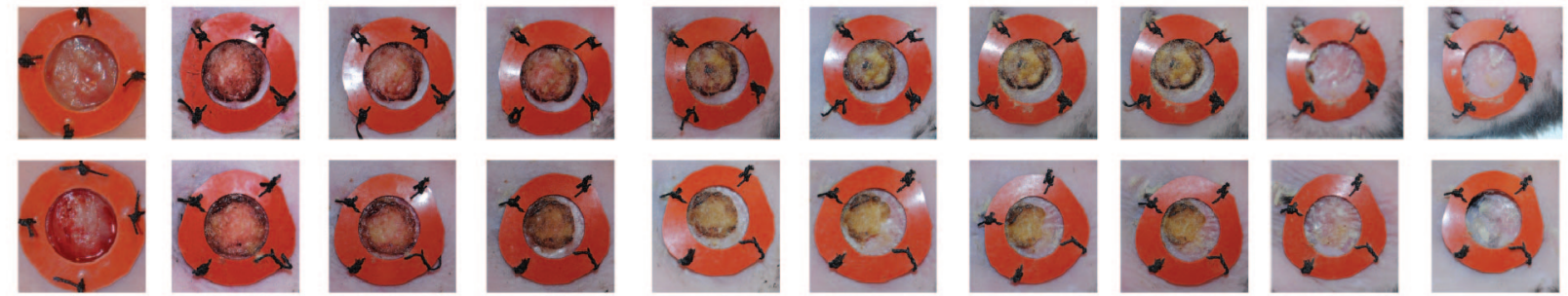

DNC: CSP: siKeap1
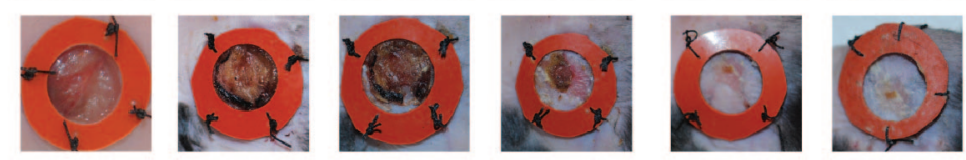

Wound

Closed
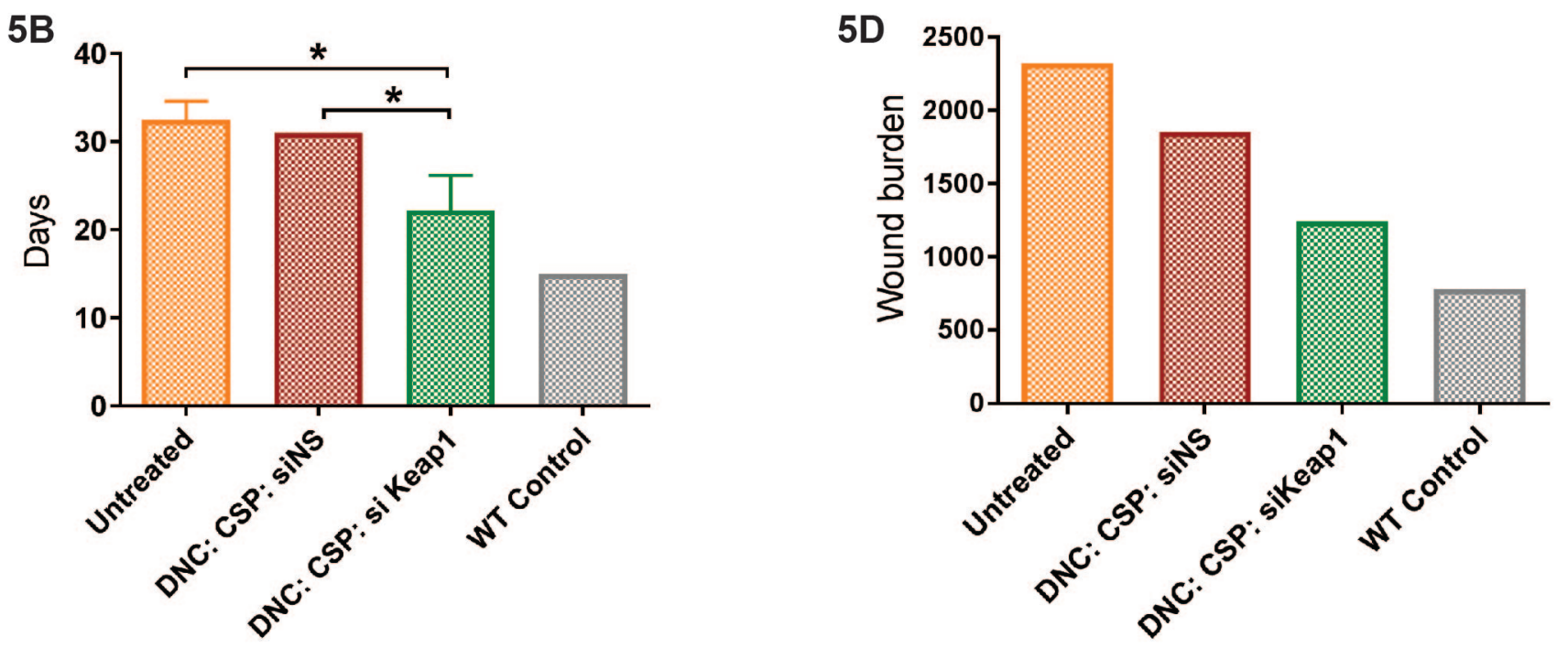

$5 C$

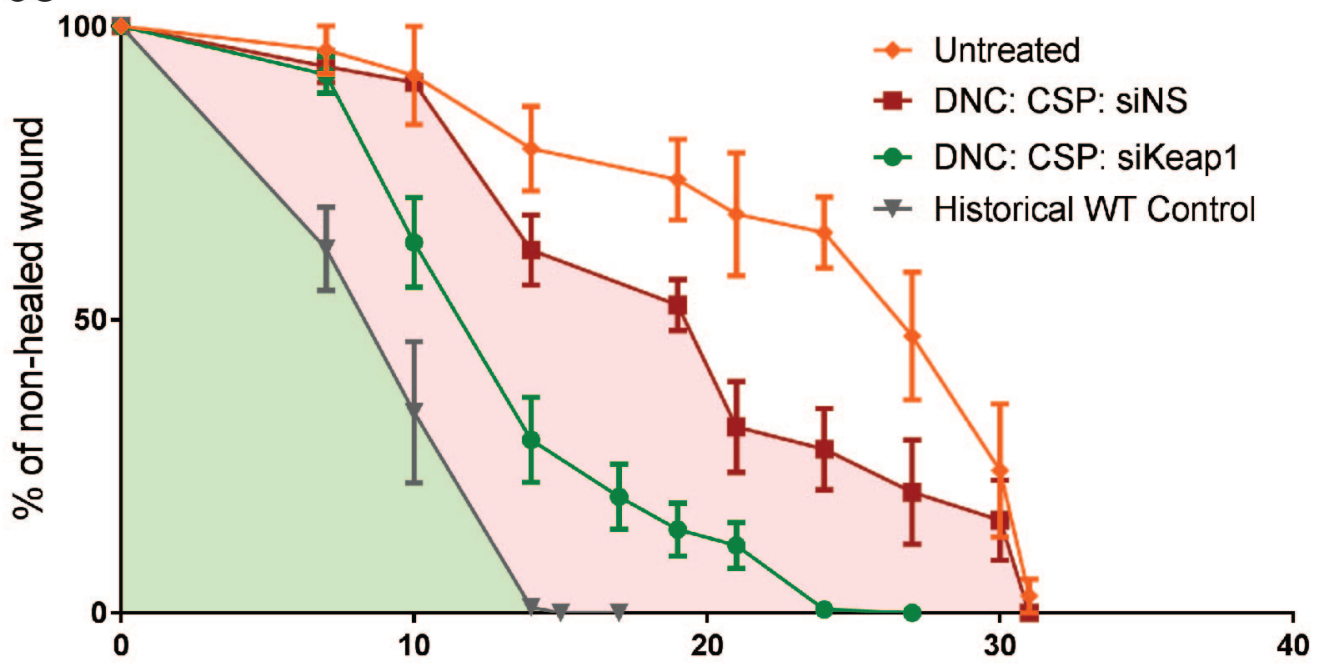




\section{$6 \mathrm{~A}$}
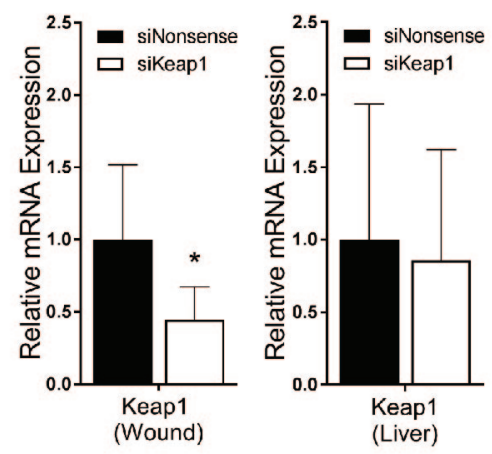

6B

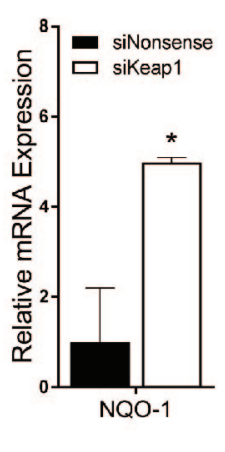

\section{$6 C$}

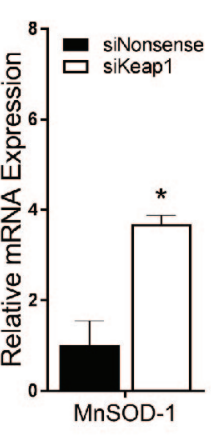

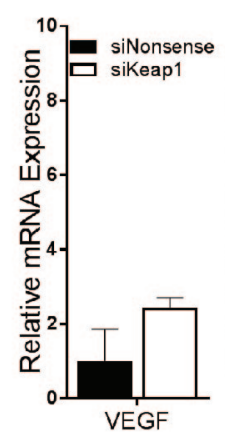
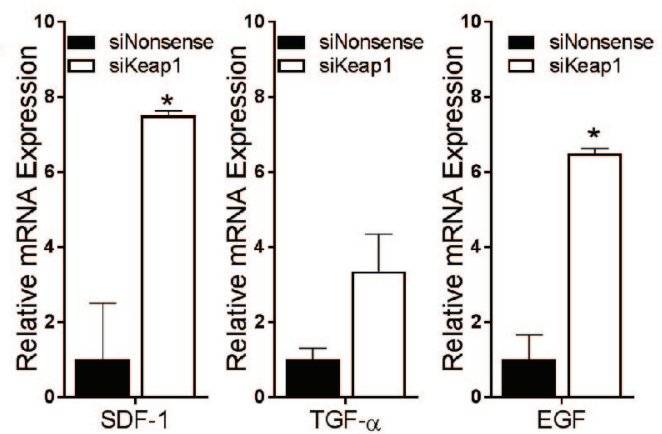

6D
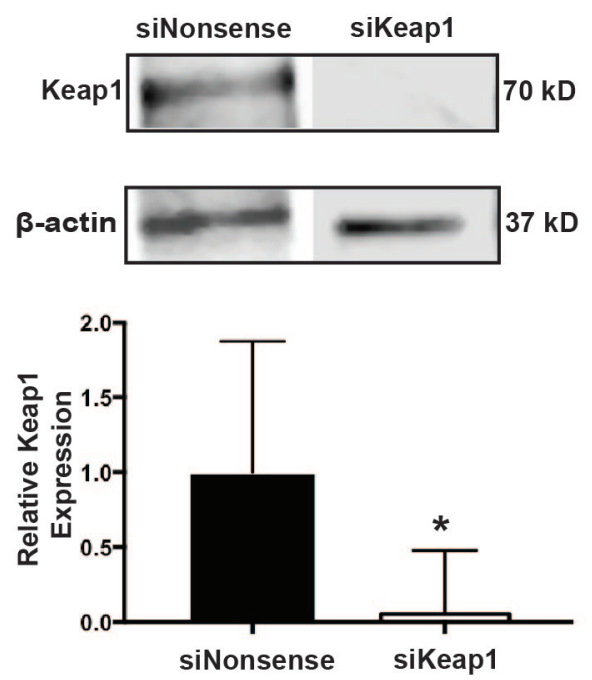

$6 F$

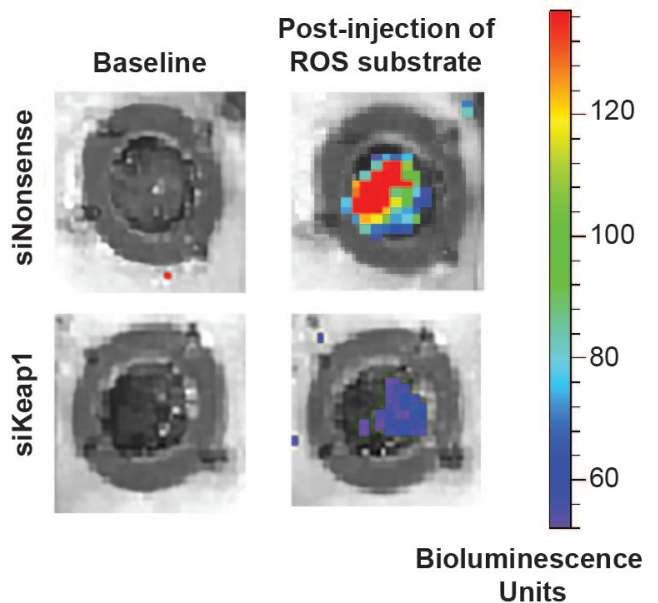

$6 \mathrm{E}$
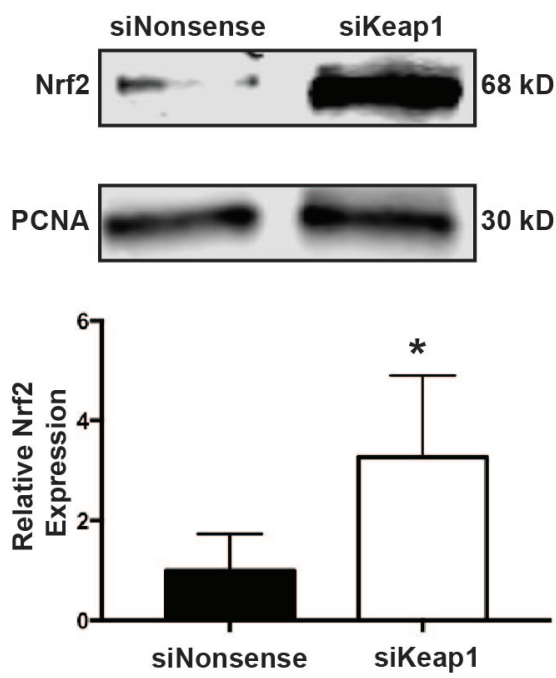

6G

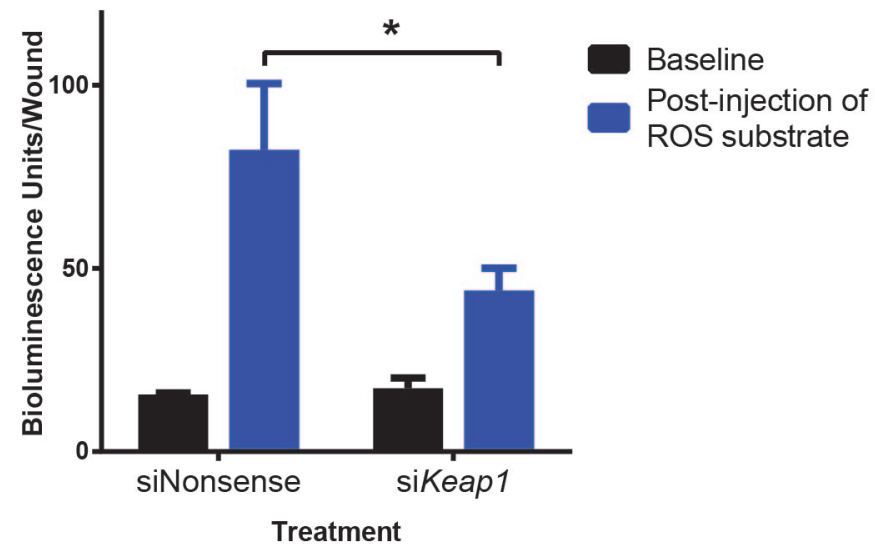


7A
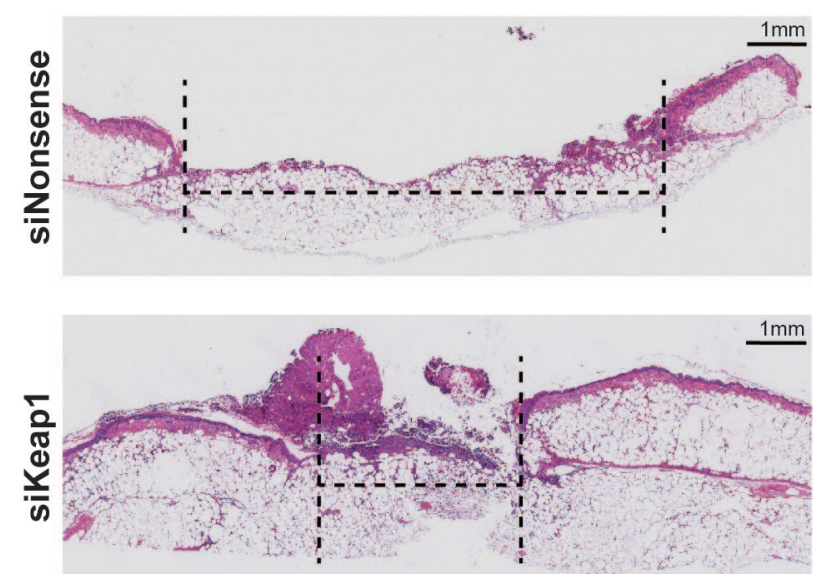

7C

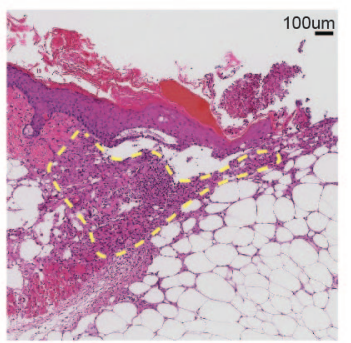

siNonsense

7E

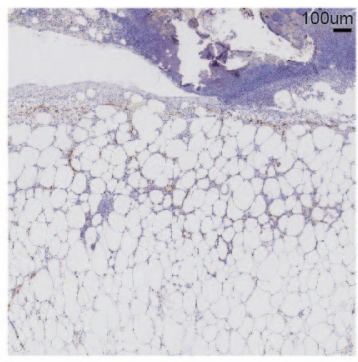

siNonsense

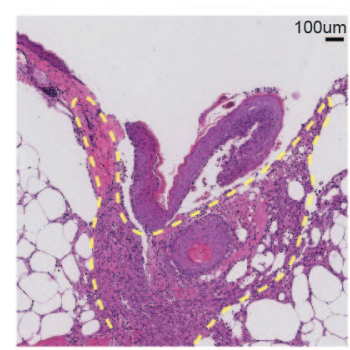

siKeap1

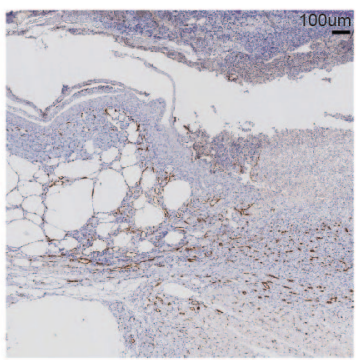

siKeap1
7B
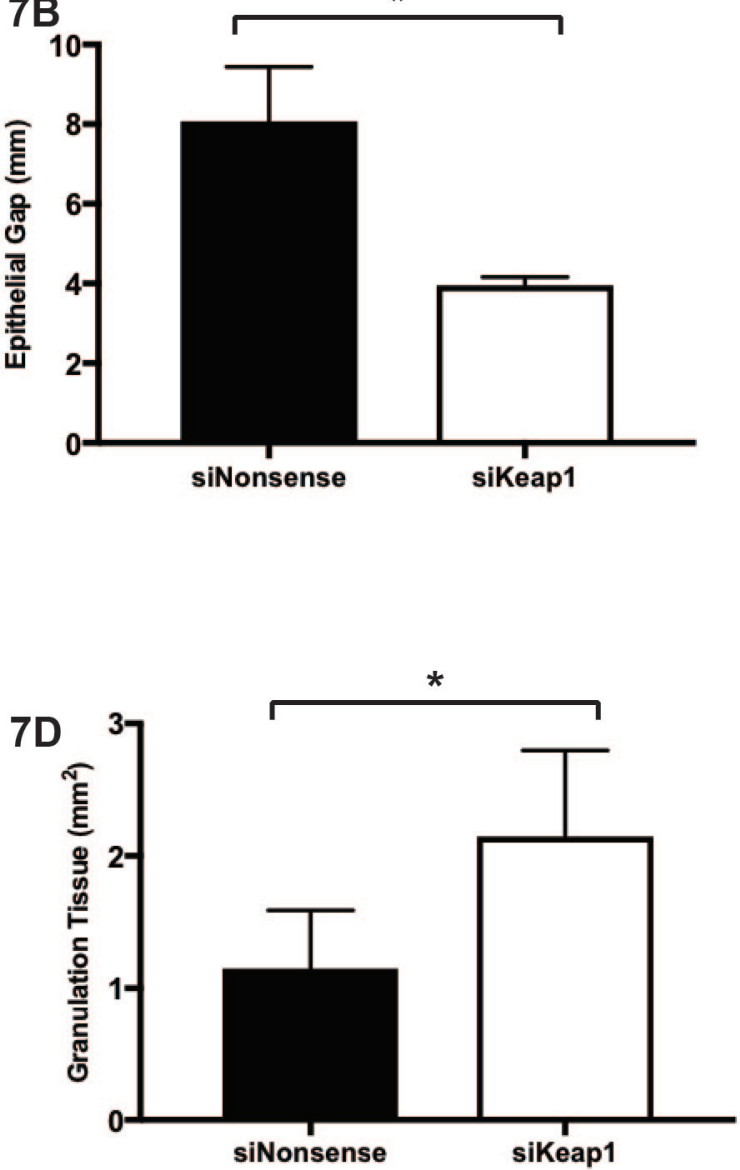

7F

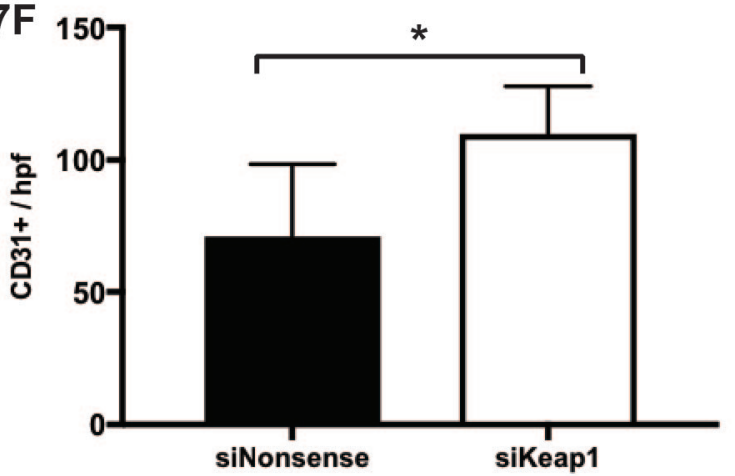

7G

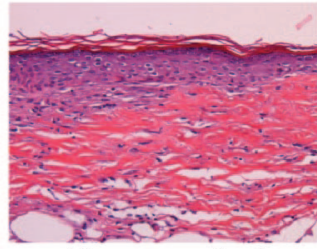

Unwounded

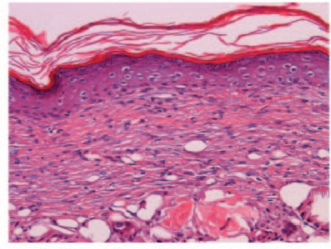

Lipofectamine

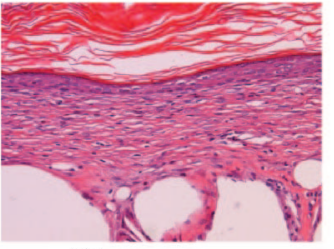

siNonsense

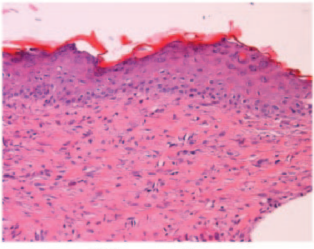

siKeap1 


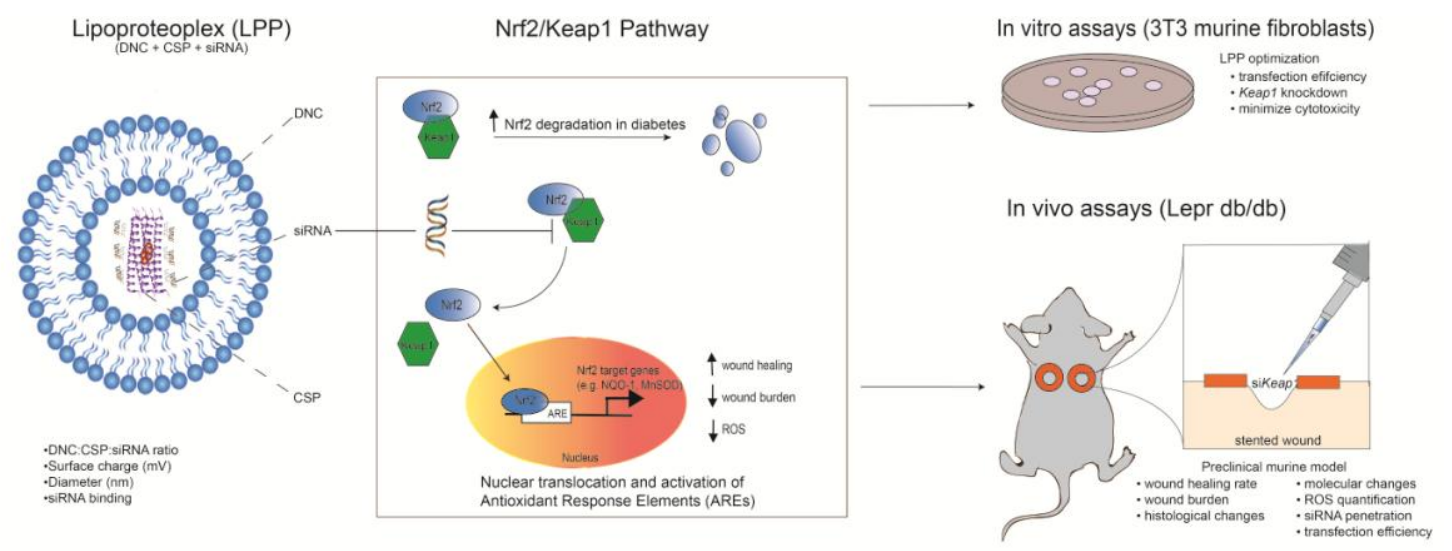

